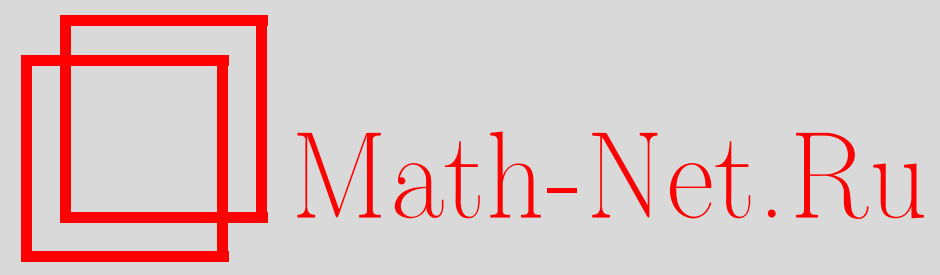

V. Bentkus, F. Götze, A. Yu. Zaitsev, Approximation of quadratic forms of independent random vectors by accompanying laws, Теория вероятн. и ее примен., 1997, том 42, выпуск 2, 308-335

DOI: https://doi.org/10.4213/tvp1805

Использование Общероссийского математического портала Math-Net.Ru подразумевает, что вы прочитали и согласны с пользовательским соглашением http://www . mathnet.ru/rus/agreement

Параметры загрузки:

IP : 54.198 .67 .100

26 апреля 2023 г., 18:05:33

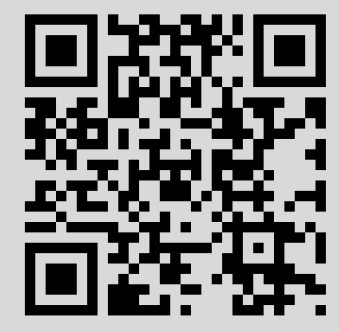


(C) 1997 r. BENTKUS V.*, GÖTZE F. ${ }^{*}$, ZAITSEV A. YU. ${ }^{* *}$

\section{APPROXIMATION OF QUADRATIC FORMS OF INDEPENDENT RANDOM VECTORS BY ACCOMPANYING LAWS ${ }^{1)}$}

Пусть $X, X_{1}, X_{2}, \ldots$ - независимые одинаково распределенные (н.о.р.) случайные векторы, принимаюшие значения из $\mathbf{R}^{d}$. Предположим, что $\mathbf{E} X=0, \mathbf{E}|X|^{8 / 3}<\infty$ и вектор $X$ не сконцентрирован в собственном подпростанстве $\mathrm{R}^{d}$. Обозначим через $Y, Y_{1}, Y_{2}, \ldots$ н.о.p. случайные векторы, имеюшие общее распределение, сопровождающее распределение $X$. В данной работе сравниваются распределения невырожденных квадратичных форм $Q\left[S_{N}\right]$ и $Q\left[T_{N}\right]$ нормированных сумм $S_{N}=N^{-1 / 2}\left(X_{1}+\cdots+X_{N}\right)$ и $T_{N}=N^{-1 / 2}\left(Y_{1}+\cdots+Y_{N}\right)$ и доказывается, что

$$
\begin{gathered}
\sup _{x}\left|\mathbf{P}\left\{Q\left[S_{N}-a\right]<x\right\}-\mathbf{P}\left\{Q\left[T_{N}-a\right]<x\right\}\right| \\
=O\left(\left(1+|a|^{4}\right) N^{-1}\right), \quad a \in \mathbf{R}^{d},
\end{gathered}
$$

при условии, что $9 \leqslant d \leqslant \infty$. Константа в этой оценке зависит от $\mathbf{E}|X|^{8 / 3}, Q$ и от ковариационного оператора $X$. Также устанавливается, что оценка $O\left(N^{-1}\right)$ является оптимальной.

Ключевые слова и фразы: сложная пуассоновская аппроксимапия, сопровождающие законы, скорости сходимости, многомерные пространства, гильбертово пространство, квадратичные формы, эллипсоид, гиперболоид.

\section{Introduction}

In the limit theory for sums of independent identically distributed (i.i.d.) random variables and vectors main efforts were devoted to study the rate

*Fakultät für Mathematik, Universität Bielefeld, Postfach 100131, 33501 Bielefeld 1 , Germany.

** Санкт-Петербургское отделение математического института РАН, Фонтанка 27, 191011 Санкт-Петербург, Россия.

1) Research supported by the SFB 343 in Bielefeld, by grants INTAS-94-4194 and INTAS-RFBR 95-0099 and by grant RFBR-DFG 96-01-00096-ge.

Research supported by the Alexander von Humboldt Foundation, by the Russian Fund of Fundamental Investigations, grant 96-01-00672, by grant RFBR-DFG 96-0100096-ge, and by the SFB 343 in Bielefeld. 
of approximation in the Central Limit Theorem (CLT) and for problems with stable limit distributions. Compared to the general structure of limit distributions of sums these are still rather special situations. Moreover, in many problems the rate of normal approximation is too slow and one needs to enlarge the class of approximating distributions. A natural approximation for the distributions of sums of a large number of i.i.d. random vectors is given by infinitely divisible (in particular, accompanying) distributions.

The problem of the estimation of the rate of infinitely divisible approximations for the distribution functions of sums of $N$ i.i.d. one-dimensional random variables as $N \rightarrow \infty$ was stated by Kolmogorov [13]. Prokhorov [20] showed that these distribution functions are approximable by the set of infinitely divisible laws in the sense of the uniform distance between distribution functions. Kolmogorov [14] showed that the rate of this approximation is uniform with respect to the class of all one-dimensional distributions and proved a rate $c N^{-1 / 5}$, where $c$ is an absolute constant. The best possible rate $c N^{-2 / 3}$ was obtained by Arak [3], [4]. For accompanying approximating distributions, the optimal bound $c N^{-1 / 3}$ was proved by Le Cam [15]. This bound was sharpened up to $c N^{-1 / 2}$ for symmetrically distributed summands by Zaitsev [21] (see also [12]) and up to $c N^{-1}$ by Arak [2] for summands with non-negative characteristic functions, see also [10]. A more detailed review of one-dimensional results mentioned above and detailed proofs can be found in the monograph of Arak and Zaitsev [5].

The majority of the corresponding multidimensional results used the uniform distance between distribution functions. Presman [19] proved an analog of Le Cam's result with a constant depending on the dimension $d<\infty$ only. When the distribution of the summands is symmetric or even has a non-negative characteristic function, the corresponding analogs of the onedimensional results of Zaitsev [21] and Arak [2] were obtained by Zaitsev [22], [23]. These results can easily be extended to the comparison of distributions on convex polytopes (see [24]). The constants in these bounds depend on certain characteristics of the polytopes what does not allow one to obtain estimates for arbitrary convex sets and balls by passing to the limit.

In spite of the fact that the results described above give the definitive answer to the question about the asymptotic behaviour of the bounds which are uniform with respect to the class of all one-dimensional or $d$ dimensional distributions, these inequalities do not describe the asymptotics of the rate of infinitely divisible approximation of $N$-fold convolutions for fixed distribution of summands. The analog of the result of Prokhorov [20] mentioned above for finite dimensional convex sets was obtained by Bakštys and Paulauskas [6]. For the approximation by accompanying laws they also proved an estimate for the Prokhorov distance between suitably normalized vectors in the infinite dimensional case which is of order $O\left(N^{-1 / 6}\right)$ in the case of Hilbert space valued vectors. Nagaev [17] proved that if $4 \leqslant d \leqslant \infty$, the norm of the summands has finite second moment and their common 
distribution is non-degenerate, then the rate of approximation by accompanying laws for balls is $O\left(N^{-1 / 2}\right)$. From the recent estimate in the CLT of Bentkus and Götze [9] it follows that this rate of approximation is $O\left(N^{-1}\right)$ for arbitrary ellipsoids and hyperboloids when $9 \leqslant d \leqslant \infty$ and the summands have non-degenerate distribution and their norm has a finite fourth moment.

In this paper we investigate the rate of approximation of the distribution of the sum of i.i.d. random vectors by the accompanying laws on the balls, ellipsoids and hyperboloids in spaces of sufficiently large dimension assuming the existence of a finite moment of order $\frac{8}{3}$ for the norm of the summands. In particular, we show that this rate of approximation is $O\left(N^{-1}\right)$ provided $9 \leqslant d \leqslant \infty$.

Let us introduce the necessary notation. Let $\mathbf{R}^{d}$ denote the $d$-dimensional space of real vectors $x=\left(x_{1}, \ldots, x_{d}\right), y=\left(y_{1}, \ldots, y_{d}\right)$ with scalar product $\langle\cdot, \cdot\rangle$ and norm $|\cdot|$ defined by $\langle x, y\rangle=x_{1} y_{1}+\cdots+x_{d} y_{d},|x|^{2}=\langle x, x\rangle$. Since our results are independent of the dimension (provided $d \geqslant 9$ ), it will be convenient to denote by $\mathbf{R}^{\infty}$ a real separable Hilbert space. Thus, $\mathbf{R}^{\infty}$ consists of all real sequences $x=\left(x_{1}, x_{2}, \ldots\right)$ such that $|x|^{2}=x_{1}^{2}+x_{2}^{2}+\cdots<\infty$.

Let $\mathfrak{F}_{d}$ be the set of probability distributions defined on the Borel $\sigma$ field of subsets of $\mathbf{R}^{d}, \mathfrak{D}_{d} \subset \mathfrak{F}_{d}$ the set of infinitely divisible laws, $E_{a} \in \mathfrak{F}_{d}$ the distribution concentrated at a point $a \in \mathbf{R}^{d}, E=E_{0} ; \mathcal{L}(\zeta) \in \mathfrak{F}_{d}$ and $\operatorname{cov} \zeta$ the distribution and the covariance operator of a random vector $\zeta \in \mathbf{R}^{d}, I\{A\}$ the indicator function of an event $A$. For $H_{1}, H_{2} \in \mathfrak{F}_{d}$, $x \in \mathbf{R}^{d}$ we denote the corresponding distribution functions by $H_{1}(x), H_{2}(x)$; $\rho\left(H_{1}, H_{2}\right) \stackrel{\text { def }}{=} \sup _{x}\left|H_{1}(x)-H_{2}(x)\right|$. Products $H_{1} H_{2}=H_{1} * H_{2}$ and powers $H^{m}=H^{m *}, H^{0}=E$ of (signed) measures should be understood in the convolution sense. By $c(\cdot), c_{1}(\cdot), c_{2}(\cdot), \ldots$ we shall denote positive quantities depending on the argument only. The symbols $c, c_{1}, c_{2}, \ldots$ will denote absolute positive constants. Notice that $c(\cdot), c$ may denote different quantities in different (or even in the same) formulas. By $\lceil\alpha\rceil$ we shall denote the integer part of a number $\alpha$. Usually we shall assume that all random variables and vectors are independent if the contrary is not clear from the context.

Let $\Xi=\left\{\zeta, \zeta_{1}, \zeta_{2}, \ldots\right\}$ be a sequence of i.i.d. random vectors in $\mathbf{R}^{d}$ with $\mathcal{L}(\zeta)=H$. By $\widetilde{\zeta}$ we shall denote a symmetrization of $\zeta$, for example $\widetilde{\zeta}=\zeta-\zeta_{1}$. Let $\Pi_{\lambda}$ denote a random variable which is independent of $\Xi$ and has Poisson distribution with parameter $\lambda>0$. Then it is evident that

$$
\mathcal{L}\left(\zeta_{1}+\cdots+\zeta_{\Pi_{\lambda}}\right)=e(\lambda H) \stackrel{\text { def }}{=} e^{-\lambda} \sum_{m=0}^{\infty} \frac{\lambda^{m} H^{m}}{m !} \in \mathfrak{D}_{d} .
$$

The distribution $e(\lambda H)$ is often called compound Poisson. It can easily be verified that, for $H_{1}, H_{2} \in \mathfrak{F}_{d}$ and $\lambda_{1}, \lambda_{2}>0$,

$$
e\left(\lambda_{1} H_{1}\right) e\left(\lambda_{2} H_{2}\right)=e\left(\left(\lambda_{1}+\lambda_{2}\right) H\right),
$$


where

$$
H=\frac{\lambda_{1}}{\lambda_{1}+\lambda_{2}} H_{1}+\frac{\lambda_{2}}{\lambda_{1}+\lambda_{2}} H_{2} \in \mathfrak{F}_{d} .
$$

In particular, for $H \in \mathfrak{F}_{d}$ and $m=2,3, \ldots$,

$$
(e(H))^{m}=e(m H)
$$

For $H \in \mathfrak{F}_{d}$ and $N=1,2, \ldots$, the set of accompanying laws is defined by

$$
\mathcal{A}_{d, N}(H)=\left\{E_{N b} e\left(N H E_{-b}\right): b \in \mathbf{R}^{d}\right\} \subset \mathfrak{D}_{d}
$$

When we approximate distributions of sums of i.i.d. summands by accompanying laws, the shift $b$ is usually the mean of truncated summands. In the case when our summands have mean zero, it is natural to consider the accompanying laws «without shift»: $D_{N}(H)=e(N H) \in \mathcal{A}_{d, N}(H)$.

Let us formulate the main result of this paper.

Let $\left\{X, X_{1}, X_{2}, \ldots\right\}$ and $\left\{Y, Y_{1}, Y_{2}, \ldots\right\}$ be two sequences of i.i.d. random vectors taking values in $\mathbf{R}^{d}$ and such that $\mathcal{L}(Y)=e(\mathcal{L}(X))$. If $\mathbf{E} X=0$ and $\mathrm{E}|X|^{2}<\infty$, then the distributions of sums

$$
S_{N}=N^{-1 / 2}\left(X_{1}+\cdots+X_{N}\right), \quad T_{N}=N^{-1 / 2}\left(Y_{1}+\cdots+Y_{N}\right)
$$

converge weakly to the distribution of a Gaussian random variable, say $\eta$, such that, according to (1.1),

$$
\mathbf{E} Y=\mathbf{E} X=\mathbf{E} \eta=0, \quad \operatorname{cov} Y=\operatorname{cov} X=\operatorname{cov} \eta .
$$

We shall assume that $\eta$ (or, equivalently, $X$ ) is not concentrated on a subspace of $\mathbf{R}^{d}$, i.e., that $\mathbf{P}\{\eta \in \mathbf{R}\}<1$ whenever $\mathbf{R} \subset \mathbf{R}^{d}$ is a proper closed linear subspace of $\mathbf{R}^{d}$.

According to (1.1), (1.4),

$$
\mathcal{L}\left(T_{N}\right)=e\left(N \mathcal{L}\left(\frac{X}{\sqrt{N}}\right)\right)=\mathcal{L}\left(N^{-1 / 2}\left(X_{1}+\cdots+X_{\Pi_{N}}\right)\right),
$$

where the random variable $\Pi_{N}$ has a Poisson distribution with parameter $N$ and is independent of $\left\{X_{1}, X_{2}, \ldots\right\}$.

For a linear symmetric and bounded operator $Q: x \mapsto Q x$ mapping $\mathbf{R}^{d}$ into $\mathbf{R}^{d}$, define the quadratic form

$$
Q[x]=\langle Q x, x\rangle .
$$

Assume that the operator $Q$ is non-degenerate, i.e., that $\operatorname{ker} Q=\{0\}$.

Theorem 1.1. Assume that $9 \leqslant d \leqslant \infty$. Let $\mathrm{E} X=0$, and $\mathbf{E}|X|^{8 / 3}<\infty$. Then

$$
\sup _{x}\left|\mathbf{P}\left\{Q\left[S_{N}-a\right]<x\right\}-\mathbf{P}\left\{Q\left[T_{N}-a\right]<x\right\}\right|=O\left(\left(1+|a|^{4}\right) N^{-1}\right) .
$$


The constant in this bound depends on $\mathrm{E}|X|^{8 / 3}$, on $Q$ and on the covariance operator of $X$ only.

A bound for constant in (1.10) under additional conditions described at the beginning of Section 3 is given in (3.31). In a particular case this constant can be estimated by an expression which depends on $Q$ and $\operatorname{cov} X$ in a more explicit form (see Theorem 4.1, inequality (4.2)).

Theorem 1.1 is formulated for normalized sums $S_{N}$ and $T_{N}$. One can easily derive from it the corresponding statement for non-normalized sums $X_{1}+\cdots+X_{n}$ and $Y_{1}+\cdots+Y_{N}$.

In the case when $Q=I$ the relation (1.10) turns into

$$
\sup _{x}\left|\mathbf{P}\left\{\left|S_{N}-a\right|^{2}<x\right\}-\mathbf{P}\left\{\left|T_{N}-a\right|^{2}<x\right\}\right|=O\left(\left(1+|a|^{4}\right) N^{-1}\right) .
$$

This bound is non-uniform with respect to $a$ and the constants will depend on the distribution of summands. This is essential since otherwise, varying $a$ or $\mathcal{L}(X)$, one could derive from (1.11) the estimate $\rho\left(\mathcal{L}\left(S_{N}\right), \mathcal{L}\left(T_{N}\right)\right)=O\left(N^{-1}\right)$ in dimension one which is evidently wrong because for the one-dimensional symmetric Bernoulli scheme with $\mathbf{P}\{X=1\}=\mathbf{P}\{X=-1\}=\frac{1}{2}$ we have $\rho\left(\mathcal{L}\left(S_{N}\right), \mathcal{L}\left(T_{N}\right)\right) \geqslant c N^{-1 / 2}$.

On the other hand, the rate of convergence in (1.10), (1.11), (4.2) cannot be faster. Consider, for example, the case when $d<\infty$ is odd, $Q=I, a=0$, $\mathbf{P}\{X=x\}=2^{-d}$ for all $x=\left(x_{1}, \ldots, x_{d}\right)$ such that $x_{j}= \pm 1, j=1, \ldots, d$.

Denote by $\mathbf{Z}_{1}=\{1,3,5, \ldots\}$ and $\mathbf{Z}_{2}=\{0,2,4, \ldots\}$ the sets of odd and even non-negative integers respectively. It is not difficult to check that

$$
\mathbf{P}\left\{\left|S_{N}\right|^{2} \in \frac{\mathbf{Z}_{1}}{N}\right\}=1-\mathbf{P}\left\{\left|S_{N}\right|^{2} \in \frac{\mathbf{Z}_{2}}{N}\right\}= \begin{cases}1, & \text { for } N \text { odd, } \\ 0, & \text { for } N \text { even. }\end{cases}
$$

From (1.8), we infer

$$
\mathbf{P}\left\{\left|T_{N}\right|^{2} \in \frac{\mathbf{Z}_{k}}{N}\right\}=\mathbf{P}\left\{\Pi_{N} \in \mathbf{Z}_{k}\right\}, \quad k=1,2
$$

Using the Local Limit Theorem, see, e.g., [18, Ch. VII, Theorem 13], it can easily be verified that

$$
\mathbf{P}\left\{\Pi_{N} \in \mathbf{Z}_{k}\right\} \underset{N \rightarrow \infty}{\longrightarrow} \frac{1}{2}, \quad k=1,2 .
$$

Choose $c_{1}(d)$ so that $\mathbf{P}\left\{|\eta|^{2} \leqslant c_{1}(d)\right\}=\frac{5}{6}$. By the CLT,

$$
\mathbf{P}\left\{\left|T_{N}\right|^{2} \leqslant c_{1}(d)\right\} \underset{N \rightarrow \infty}{\longrightarrow} \frac{5}{6} .
$$

According to (1.13)-(1.15), for sufficiently large $N$ we have

$$
\mathbf{P}\left\{\left|T_{N}\right|^{2} \in \frac{\mathbf{Z}_{k}}{N},\left|T_{N}\right|^{2} \leqslant c_{1}(d)\right\} \geqslant \frac{1}{4}, \quad k=1,2 .
$$


It is clear that (1.16) implies that

$$
\max \left\{\mathbf{P}\left\{\left|T_{N}\right|^{2}=\frac{l}{N}\right\}: l \in \mathbb{Z}_{k}, l \leqslant c_{1}(d) N\right\} \geqslant \frac{c_{2}(d)}{N}, \quad k=1,2 .
$$

From (1.12), (1.17) it follows that

$$
\sup _{x}\left|\mathbf{P}\left\{\left|S_{N}\right|^{2}<x\right\}-\mathbf{P}\left\{\left|T_{N}\right|^{2}<x\right\}\right| \geqslant \frac{c_{2}(d)}{2 N} .
$$

For even $d$ a similar example can easily be constructed too. It suffices to add to $X$ in the previous example an independent $(d+1)$-th coordinate taking values \pm 2 with probability $\frac{1}{2}$ which allows one to repeat the arguments above.

If $\mathbf{E}|X|^{4}<\infty$, the bound of the form $O\left(N^{-1}\right)$ in (1.10) follows from an estimate in the CLT, contained in the main result of Bentkus and Götze [9]. Theorem 1.1 shows that this rate of approximation remains valid given weaker moment assumptions where the Gaussian approximation is essentially slower. Thus, Theorem 1.1 can be considered as an analog of a result of Zaitsev [25]. He proved that if $d=1, \mathbf{E} X=0$, and $\mathbf{E}|X|^{3 / 2}<\infty$, then $\rho\left(\mathcal{L}\left(S_{N}\right), \mathcal{L}\left(T_{N}\right)\right)=O\left(N^{-1 / 2}\right)$.

For the proof of Theorems 1.1 and 4.1 we use an analog of the well known one-dimensional Esseen bound which estimates the uniform distance $\rho(\cdot, \cdot)$ in terms of characteristic functions (see Lemma 2.6). For sufficiently large values of $|t|$, provided that $O\left(N^{2 / 9}\right) \leqslant|t| \leqslant O(N)$ we estimate the integrals of characteristic functions with the help of recent results of Bentkus and Götze [8], [9] (see Lemmas 2.1 and 2.2). For $|t| \leqslant O\left(N^{2 / 9}\right.$ ) we use an expansion of characteristic functions contained in Lemma 2.5. Besides, we use a truncation and the standard techniques of estimation in Hilbert spaces.

\section{A uxiliary results}

Let us define the following classes of probability distributions which will appear in our arguments. Let $s \leqslant d$ be a positive integer number and $0<r \leqslant s^{-1}, 0<\delta \leqslant \frac{1}{2}$. Assume that $P: \mathbf{R}^{d} \rightarrow \mathbf{R}^{d}$ is a linear operator and $y_{1}, \ldots, y_{s} \in \mathbf{R}^{d}$ an orthonormal system of vectors. Let $\varepsilon_{1}, \varepsilon_{2}, \ldots$ denote i.i.d. symmetric Rademacher random variables.

We say that a distribution $\mathcal{L}(\zeta) \in \mathfrak{F}_{d}$ belongs to the class $\mathcal{N}(s, r, \delta, P$; $\left.y_{1}, \ldots, y_{s}\right)$ if

$$
\min \left\{\min _{1 \leqslant j \leqslant s} \mathbf{P}\left\{\left|\zeta-y_{j}\right| \leqslant \delta\right\}, \min _{1 \leqslant j \leqslant s} \mathbf{P}\left\{\left|\zeta-P y_{j}\right| \leqslant \delta\right\}\right\} \geqslant r
$$

We say that the distribution of a discrete random vector $W \in \mathbf{R}^{d}$ belongs to the class $\Gamma\left(s, \delta ; y_{1}, \ldots, y_{s}\right)$ if $W$ is distributed as $\varepsilon_{1} w_{1}+\cdots+\varepsilon_{s} w_{s}$, with some (non-random) $w_{1}, \ldots, w_{s} \in \mathbf{R}^{d}$ such that $\left|w_{j}-y_{j}\right| \leqslant \delta$, for all $j=1, \ldots, s$. 
Introduce the function $M_{s}(v, w), v \in \mathbf{R}, w>0$, by

$$
M_{s}(v, w)=\left\{\begin{array}{lll}
|v|^{-s / 2}, & \text { for } & |v| \leqslant \sqrt{w} \\
\left(\frac{|v|}{w}\right)^{s / 2}, & \text { for } & |v| \geqslant \sqrt{w}
\end{array}\right.
$$

Notice that, for all $v \in \mathbf{R}, w>0$,

$$
c_{3}(s)\left(|v|^{-s / 2}+\left(\frac{|v|}{w}\right)^{s / 2}\right) \leqslant M_{s}(v, w) \leqslant c_{4}(s)\left(|v|^{-s / 2}+\left(\frac{|v|}{w}\right)^{s / 2}\right) .
$$

It is clear that $(2.2),(2.3)$ imply that

$$
\text { if } \quad c_{5}(s)\left|v_{0}\right| \leqslant|v| \leqslant c_{6}(s)\left|v_{0}\right|, \quad 0 \leqslant c_{7}(s) w_{0} \leqslant w \leqslant c_{8}(s) w_{0}
$$

then $\quad c_{9}(s) M_{s}\left(v_{0}, w_{0}\right) \leqslant M_{s}(v, w) \leqslant c_{10}(s) M_{s}\left(v_{0}, w_{0}\right)$.

In the proof of Theorem 1.1 we shall use the following Lemmas 2.1-2.6 in which $Q$ denotes the symmetric bounded operator defined in Section 1.

Lemma 2.1 (Bentkus and Götze [9, Corollary 5.5 and Theorems 6.3, 7.1]). Let $s$ be a positive integer number, $1 \leqslant s \leqslant d, 0<r \leqslant s^{-1}, 0<\delta \leqslant \frac{1}{2}$. Suppose that the operator $Q$ is isometric. Assume that the symmetrization $\widetilde{Z}^{*}$ of a random vector $Z^{*} \in \mathbf{R}^{d}$, satisfies

$$
\mathcal{L}\left(\widetilde{Z}^{*}\right) \in \mathcal{N}\left(s, r, \delta, Q ; y_{1}, \ldots, y_{s}\right)
$$

for an orthonormal system of vectors $y_{1}, \ldots, y_{s} \in \mathbf{R}^{d}$. Let $n$ be a positive integer number,

$$
\Upsilon_{n}^{*}=Z_{1}^{*}+\cdots+Z_{n}^{*},
$$

where $Z_{1}^{*}, Z_{2}^{*}, \ldots$ denote independent copies of $Z^{*}$. Let $l=\lceil r n /(4 s)\rceil \geqslant 1$, $0<A \leqslant B$. Then

$$
\sup _{z \in \mathbf{R}^{d}} \int_{A \leqslant|t| \leqslant B}\left|\mathbf{E} \exp \left\{2 i t Q\left[\Upsilon_{n}^{*}-z\right]\right\}\right| \frac{d t}{|t|} \leqslant J+c(s)(r n)^{-2} \log \frac{B}{A},
$$

where

$$
\begin{aligned}
J & =\sup _{\Lambda} \sup _{z \in \mathbf{R}^{d}} \int_{A \leqslant|t| \leqslant B} \sqrt{\varphi_{\Lambda}(z, 4 t l)} \frac{d t}{|t|}, \\
\varphi_{\Lambda}(z, u) & =\left|\mathbf{E} \exp \left\{i u l^{-1} Q\left[\Lambda_{l}+Q \Lambda_{l}^{\prime}-z\right]\right\}\right|^{2},
\end{aligned}
$$

and the supremum $\sup _{\Lambda}$ is taken over all random vectors $\Lambda_{l}$ and $\Lambda_{l}^{\prime}$ of type

$$
\Lambda_{l}=W_{1}+\cdots+W_{l}, \quad \Lambda_{l}^{\prime}=W_{1}^{\prime}+\cdots+W_{l}^{\prime},
$$

where $W_{1}, \ldots, W_{l}, W_{1}^{\prime}, \ldots, W_{l}^{\prime} \in \mathbf{R}^{d}$ denote independent (generally speaking, non-identically distributed) random vectors with distributions belonging to the class $\Gamma\left(s, \delta ; y_{1}, \ldots, y_{s}\right)$. 
If we additionally assume that $\delta=\frac{1}{50 s}$, then, for all $\gamma \geqslant 0, u \in \mathbf{R}$, we have

$$
\varphi_{\Lambda}(z, u+\gamma) \varphi_{\Lambda}(z, u-\gamma) \leqslant c(s) M_{s}^{2}(\gamma, l)
$$

and

$$
\sup _{z \in \mathbf{R}^{\mathbf{d}}}\left|\operatorname{E} \exp \left\{i u n^{-1} Q\left[\Upsilon_{n}^{*}-z\right]\right\}\right| \leqslant c(s) M_{s}(r u, r n) .
$$

Lemma 2.2 (Bentkus and Götze [8, Lemma 6.1]). Assume that a nonnegative function $\psi(t), t \geqslant 0$, is continuous and $\psi(0)=1$. Furthermore, suppose that there exists a number $\lambda \geqslant 1$ such that, for some $s \geqslant 9, D \geqslant 1$,

$$
\psi(t) \psi(t+\Delta) \leqslant \lambda^{2} M_{s}^{2}(\Delta, D), \quad \text { for all } t \geqslant 0 \text { and } \Delta \geqslant 0 .
$$

Then

$$
\int_{D^{(s-2) / s}}^{D} \sqrt{\psi(t)} \frac{d t}{t} \leqslant \frac{c(s) \lambda}{D} .
$$

Lemma 2.3 (Rosenthal's inequality). Let $\psi_{1}, \ldots, \psi_{\rho} \in \mathbf{R}^{d}$ be independent mean zero random vectors. Then

$$
\mathbf{E}\left|\sum_{l=1}^{\rho} \psi_{l}\right|^{\beta} \leqslant c(\beta)\left(\sum_{l=1}^{\rho} \mathbf{E}\left|\psi_{l}\right|^{\beta}+\left(\sum_{l=1}^{\rho} \mathbf{E}\left|\psi_{l}\right|^{2}\right)^{\beta / 2}\right), \quad 2 \leqslant \beta<\infty .
$$

P r o of. A result of de Acosta [1] implies

$$
\mathbf{E}\left|\sum_{l=1}^{\rho} \psi_{l}\right|^{\beta} \leqslant c(\beta)\left(\left(\mathbf{E}\left|\sum_{l=1}^{\rho} \psi_{l}\right|^{2}\right)^{\beta / 2}+\sum_{l=1}^{\rho} \mathbf{E}\left|\psi_{l}\right|^{\beta}+\left(\sum_{l=1}^{\rho} \mathbf{E}\left|\psi_{l}\right|^{2}\right)^{\beta / 2}\right)
$$

Thus, an application of the obvious identity $\mathbf{E}\left|\sum_{l=1}^{\rho} \psi_{l}\right|^{2}=\sum_{l=1}^{\rho} \mathbf{E}\left|\psi_{l}\right|^{2}$ completes the proof.

We shall need also the following auxiliary bound for the convergence rate in the CLT in finite dimensional and Hilbert spaces for expectations of smooth functions.

Lemma 2.4. Let a function $\Psi: \mathbf{R}^{d} \rightarrow \mathbf{R}$ be three times Frechét differentiable. Then there exists an absolute constant $c$ such that

$$
\left|\mathbf{E} \Psi\left(S_{m}\right)-\mathbf{E} \Psi(\eta)\right| \leqslant c \mathbf{E}|X|^{8 / 3} m^{-1 / 3}\left\|\Psi^{(2)}\right\|_{\infty}^{1 / 3}\left\|\Psi^{(3)}\right\|_{\infty}^{2 / 3},
$$

where $\left\|\Psi^{(l)}\right\|_{\infty}=\sup _{x \in \mathbf{R}^{d}}\left\|\Psi^{(l)}(x)\right\|, l=2,3$, and $\left\|\Psi^{(l)}(x)\right\|$ denotes the norm of the l-th Frechét derivative.

$\mathrm{P}$ r o o f. The result can be proved like Theorem 1.2 in [7]. For the sake of completeness we include a sketch of the proof. Without loss of generality we assume that

$$
\eta=m^{-1 / 2}\left(\eta_{1}+\cdots+\eta_{m}\right)
$$


where $\eta_{1}, \ldots, \eta_{m}$ are i.i.d. Gaussian random vectors with $\mathcal{L}\left(\eta_{j}\right)=\mathcal{L}(\eta)$. Assume also that the vectors $\left\{X_{j}, \eta_{k} ; j, k=1, \ldots, m\right\}$ are independent. Denote

$$
V_{j}=m^{-1 / 2}\left(X_{1}+\cdots+X_{j-1}+\eta_{j+1}+\cdots+\eta_{m}\right) \text {. }
$$

Then

$$
\left|\mathbf{E} \Psi\left(S_{m}\right)-\mathbf{E} \Psi(\eta)\right| \leqslant \delta_{1}+\cdots+\delta_{m}
$$

where

$$
\delta_{j}=\left|\mathbf{E} \Psi\left(V_{j}+\frac{X_{j}}{\sqrt{m}}\right)-\mathbf{E} \Psi\left(V_{j}+\frac{\eta_{j}}{\sqrt{m}}\right)\right|, \quad j=1, \ldots, m .
$$

It is clear that, by the Taylor expansions in powers of $X_{j} / \sqrt{m}$ and $\eta_{j} / \sqrt{m}$,

$$
\begin{aligned}
& \left|\Psi\left(V_{j}+\frac{X_{j}}{\sqrt{m}}\right)-\Psi\left(V_{j}+\frac{\eta_{j}}{\sqrt{m}}\right)\right| \\
& \leqslant c m^{-1}\left(\left|X_{j}\right|^{2}+\left|\eta_{j}\right|^{2}\right)\left\|\Psi^{(2)}\right\|_{\infty} \\
& \left|\Psi\left(V_{j}+\frac{X_{j}}{\sqrt{m}}\right)-\Psi\left(V_{j}+\frac{\eta_{j}}{\sqrt{m}}\right)\right| \\
& \leqslant c m^{-3 / 2}\left(\left|X_{j}\right|^{3}+\left|\eta_{j}\right|^{3}\right)\left\|\Psi^{(3)}\right\|_{\infty} .
\end{aligned}
$$

Using Lemma 2.3 and Hölder's inequality, one can easily derive that

$$
\mathbf{E}|\eta|^{8 / 3} \leqslant c\left(\mathbf{E}|\eta|^{2}\right)^{4 / 3}=c\left(\mathbf{E}|X|^{2}\right)^{4 / 3} \leqslant c \mathbf{E}|X|^{8 / 3} .
$$

Combining the estimates (2.19) and using (2.20), we see that inequality (2.14) follows from (2.17) and (2.18).

Lemma 2.5. Let $\zeta_{1}^{*}, \zeta_{2}^{*} \in \mathbf{R}^{d} ; \tau_{1}, \tau_{2} \in \mathbf{R}$ be independent random vectors and variables such that $\mathbf{E}\left|\zeta_{1}^{*}\right|^{4}<\infty, \mathbf{E}\left|\zeta_{2}^{*}\right|^{4}<\infty$. Assume that $\tau_{1}, \tau_{2}$ are uniformly distributed on the interval $[0,1]$. Let $\xi \in \mathbf{R}^{d}$ and $t \in \mathbf{R}$ be nonrandom. Write

$$
\begin{aligned}
J_{0}= & \mathbf{E} \exp \left\{i t Q\left[\xi+\zeta_{1}^{*}+\zeta_{2}^{*}\right]\right\}-\mathbf{E} \exp \left\{i t Q\left[\xi+\zeta_{1}^{*}\right]\right\} \\
& -\mathbf{E} \exp \left\{i t Q\left[\xi+\zeta_{2}^{*}\right]\right\}+\exp \{i t Q[\xi]\}
\end{aligned}
$$

and

$$
\begin{aligned}
v_{1} & =2 i t\left\langle Q \xi, \zeta_{1}^{*}\right\rangle, \quad v_{2}=i t Q\left[\zeta_{1}^{*}\right], \quad u=2 i t\left\langle Q \zeta_{1}^{*}, \zeta_{2}^{*}\right\rangle . \\
w_{1} & =2 i t\left\langle Q \xi, \zeta_{2}^{*}\right\rangle, \quad w_{2}=i t Q\left[\zeta_{2}^{*}\right], \quad
\end{aligned}
$$

Then we have

$$
J_{0}=J_{1}+J_{2}
$$

where

$$
J_{1}=\mathbf{E} v_{2} w_{2} \exp \left\{w_{1}+\tau_{1} w_{2}\right\} \exp \{i t Q[\xi]\}
$$




$$
\begin{array}{rl}
+ & \mathbf{E}\left(v_{1}+v_{2}+u\right)^{2} w_{2}\left(1-\tau_{2}\right) \\
& \times \exp \left\{w_{1}+\tau_{1} w_{2}+\tau_{2}\left(v_{1}+v_{2}+u\right)\right\} \exp \{i t Q[\xi]\} \\
& +\mathbf{E}\left(w_{1}+u\right)^{2} v_{2}\left(1-\tau_{2}\right) \exp \left\{v_{1}+\tau_{2}\left(w_{1}+u\right)+\tau_{1} v_{2}\right\} \exp \{i t Q[\xi]\} \\
& +\mathbf{E} u^{2}\left(1-\tau_{1}\right) \exp \left\{v_{1}+w_{1}+\tau_{1} u\right\} \exp \{i t Q[\xi]\} \\
& +\mathbf{E} u v_{1} w_{1} \exp \left\{\tau_{1} w_{1}\right\} \exp \{i t Q[\xi]\} \\
& +\mathbf{E} u v_{1}^{2} w_{1}\left(1-\tau_{1}\right) \exp \left\{\tau_{1} v_{1}+\tau_{2} w_{1}\right\} \exp \{i t Q[\xi]\} \\
& +\mathbf{E} v_{1}^{2} w_{1}^{2}\left(1-\tau_{1}\right)\left(1-\tau_{2}\right) \exp \left\{\tau_{2} v_{1}+\tau_{1} w_{1}\right\} \exp \{i t Q[\xi]\} \\
J_{2} & \mathbf{E}\left(v_{1}+u\right) w_{2} \exp \left\{w_{1}+\tau_{1} w_{2}\right\} \exp \{i t Q[\xi]\} \\
& +\mathbf{E}\left(w_{1}+u\right) v_{2} \exp \left\{v_{1}+\tau_{1} v_{2}\right\} \exp \{i t Q[\xi]\} \\
& +\mathbf{E}\left(u+u v_{1}\right) \exp \{i t Q[\xi]\} \\
& +\mathbf{E} u w_{1} \exp \left\{\tau_{1} w_{1}\right\} \exp \{i t Q[\xi]\} \\
& +\mathbf{E} u v_{1}^{2}\left(1-\tau_{1}\right) \exp \left\{\tau_{1} v_{1}\right\} \exp \{i t Q[\xi]\}+\mathbf{E} w_{1} v_{1} \exp \{i t Q[\xi]\} \\
& +\mathbf{E} w_{1} v_{1}^{2}\left(1-\tau_{1}\right) \exp \left\{\tau_{1} v_{1}\right\} \exp \{i t Q[\xi]\} \\
& +\mathbf{E} v_{1} w_{1}^{2}\left(1-\tau_{1}\right) \exp \left\{\tau_{1} w_{1}\right\} \exp \{i t Q[\xi]\}
\end{array}
$$

P r o of. The proof is based on the Taylor expansion with remainder term in integral form in powers of $v_{1}, v_{2}, w_{1}, w_{2}, u$. For example, using (1.9), we can use the following expansions:

$$
\begin{aligned}
& \mathbf{E} \exp \left\{i t Q\left[\xi+\zeta_{1}^{*}\right]-i t Q[\xi]\right\}=\mathbf{E} \exp \left\{v_{1}+v_{2}\right\} \\
& \quad=\mathbf{E} \exp \left\{v_{1}\right\}+\mathbf{E} v_{2} \exp \left\{v_{1}+\tau_{1} v_{2}\right\} \\
& \mathbf{E} \exp \left\{i t Q\left[\xi+\zeta_{2}^{*}\right]-i t Q[\xi]\right\}=\mathbf{E} \exp \left\{w_{1}+w_{2}\right\} \\
& \quad=\mathbf{E} \exp \left\{w_{1}\right\}+\mathbf{E} w_{2} \exp \left\{w_{1}+\tau_{1} w_{2}\right\} \\
& \mathbf{E} \exp \left\{i t Q\left[\xi+\zeta_{1}^{*}+\zeta_{2}^{*}\right]-i t Q[\xi]\right\}=\mathbf{E} \exp \left\{v_{1}+v_{2}+w_{1}+w_{2}+u\right\} \\
& \quad=\mathbf{E} \exp \left\{v_{1}+v_{2}+w_{1}+u\right\}+\mathbf{E} w_{2} \exp \left\{v_{1}+v_{2}+w_{1}+u+\tau_{1} w_{2}\right\} \\
& \mathbf{E} w_{2} \exp \left\{v_{1}+v_{2}+w_{1}+u+\tau_{1} w_{2}\right\}=\mathbf{E} w_{2} \exp \left\{w_{1}+\tau_{1} w_{2}\right\} \\
& \quad+\mathbf{E}\left(v_{1}+v_{2}+u\right) w_{2} \exp \left\{w_{1}+\tau_{1} w_{2}\right\} \\
& \quad+\mathbf{E}\left(v_{1}+v_{2}+u\right)^{2} w_{2}\left(1-\tau_{2}\right) \exp \left\{w_{1}+\tau_{1} w_{2}+\tau_{2}\left(v_{1}+v_{2}+u\right)\right\} \\
& \mathbf{E} \exp \left\{v_{1}+v_{2}+w_{1}+u\right\}=\mathbf{E} \exp \left\{v_{1}+w_{1}+u\right\} \\
& \quad+\mathbf{E} v_{2} \exp \left\{v_{1}+w_{1}+u+\tau_{1} v_{2}\right\} \\
& \mathbf{E} v_{2} \exp \left\{v_{1}+w_{1}+u+\tau_{1} v_{2}\right\}=\mathbf{E} v_{2} \exp \left\{v_{1}+\tau_{1} v_{2}\right\} \\
& \quad+\mathbf{E}\left(w_{1}+u\right) v_{2} \exp \left\{v_{1}+\tau_{1} v_{2}\right\} \\
& \quad+\mathbf{E}\left(w_{1}+u\right)^{2} v_{2}\left(1-\tau_{2}\right) \exp \left\{v_{1}+\tau_{2}\left(w_{1}+u\right)+\tau_{1} v_{2}\right\} \\
& \mathbf{E} \exp \left\{v_{1}+w_{1}+u\right\}=\mathbf{E} \exp \left\{v_{1}+w_{1}\right\}+\mathbf{E} u \exp \left\{v_{1}+w_{1}\right\} \\
& \quad+\mathbf{E} u^{2}\left(1-\tau_{1}\right) \exp \left\{v_{1}+w_{1}+\tau_{1} u\right\} \\
& \mathbf{E} u \exp \left\{v_{1}+w_{1}\right\}=\mathbf{E}\left(u+u v_{1}\right) \exp \left\{w_{1}\right\}
\end{aligned}
$$




$$
\begin{aligned}
& \quad+\mathbf{E} u v_{1}^{2}\left(1-\tau_{1}\right) \exp \left\{\tau_{1} v_{1}+w_{1}\right\} \\
& \mathbf{E}\left(u+u v_{1}\right) \exp \left\{w_{1}\right\}=\mathbf{E}\left(u+u v_{1}\right)+\mathbf{E}\left(u+u v_{1}\right) w_{1} \exp \left\{\tau_{1} w_{1}\right\} \\
& \mathbf{E} u v_{1}^{2}\left(1-\tau_{1}\right) \exp \left\{\tau_{1} v_{1}+w_{1}\right\}=\mathbf{E} u v_{1}^{2}\left(1-\tau_{1}\right) \exp \left\{\tau_{1} v_{1}\right\} \\
& \quad+\mathbf{E} u v_{1}^{2} w_{1}\left(1-\tau_{1}\right) \exp \left\{\tau_{1} v_{1}+\tau_{2} w_{1}\right\} \\
& \mathbf{E} \exp \left\{v_{1}+w_{1}\right\}=\mathbf{E} \exp \left\{v_{1}\right\}+\mathbf{E} w_{1} \exp \left\{v_{1}\right\} \\
& \quad+\mathbf{E} w_{1}^{2}\left(1-\tau_{1}\right) \exp \left\{v_{1}+\tau_{1} w_{1}\right\} \\
& \mathbf{E} \exp \left\{w_{1}\right\}=1+\mathbf{E} w_{1}+\mathbf{E} w_{1}^{2}\left(1-\tau_{1}\right) \exp \left\{\tau_{1} w_{1}\right\} \\
& \mathbf{E} w_{1} \exp \left\{v_{1}\right\}=\mathbf{E} w_{1}+\mathbf{E} w_{1} v_{1}+\mathbf{E} w_{1} v_{1}^{2}\left(1-\tau_{1}\right) \exp \left\{\tau_{1} v_{1}\right\} \\
& \mathbf{E} w_{1}^{2}\left(1-\tau_{1}\right) \exp \left\{v_{1}+\tau_{1} w_{1}\right\}=\mathbf{E}\left(w_{1}^{2}+v_{1} w_{1}^{2}\right)\left(1-\tau_{1}\right) \exp \left\{\tau_{1} w_{1}\right\} \\
& \quad+\mathbf{E} v_{1}^{2} w_{1}^{2}\left(1-\tau_{1}\right)\left(1-\tau_{2}\right) \exp \left\{\tau_{2} v_{1}+\tau_{1} w_{1}\right\}
\end{aligned}
$$

The following Lemma 2.6 can easily be derived from the results of Le Cam [15] and Esseen [11] (see also [18, Ch. V, Theorem 1; Ch. III, Lemma 3] or [5, Ch. III, Theorem 1.2, Ch. II, Lemma 1.4]).

Lemma 2.6. Let $H_{1}, H_{2}$ be one-dimensional probability distributions with characteristic functions $h_{1}(\cdot), h_{2}(\cdot)$. Then, for any $T>0$,

$$
\begin{aligned}
\rho\left(H_{1}, H_{2}\right) \leqslant & c \int_{0}^{T}\left|\frac{h_{1}(t)-h_{2}(t)}{t}\right| d t \\
& +\frac{c}{T} \min \left\{\int_{0}^{T}\left|h_{1}(t)\right| d t, \int_{0}^{T}\left|h_{2}(t)\right| d t\right\} .
\end{aligned}
$$

The expectation $\mathbf{E}_{\mu}$ with respect to a random vector (or variable) $\mu$ we define as the conditional expectation

$$
\mathbf{E}_{\mu} f(\mu, \nu, \xi, \ldots)=\mathbf{E}\{f(\mu, \nu, \xi \ldots) \mid \nu, \xi, \ldots\}
$$

given all random objects except $\mu$. Below we shall use that if the random vectors $\mu \in \mathbf{R}^{d}, \nu$ are independent and $f(\cdot, \cdot), g(\cdot, \cdot, \cdot)$ are measurable functions (possibly, complex valued), then

$$
|\mathbf{E} f(\mu, \nu)|=\left|\mathbf{E}\left(\mathbf{E}_{\nu} f(\mu, \nu)\right)\right| \leqslant \mathbf{E}\left|\mathbf{E}_{\nu} f(\mu, \nu)\right| \leqslant \sup _{z \in \mathbf{R}^{d}}|\mathbf{E} f(z, \nu)|
$$

and, analogously,

$$
\begin{aligned}
\int|\mathbf{E} g(t, \mu, \nu)| d t & \leqslant \int \mathbf{E}\left|\mathbf{E}_{\nu} g(t, \mu, \nu)\right| d t=\mathbf{E} \int\left|\mathbf{E}_{\nu} g(t, \mu, \nu)\right| d t \\
& \leqslant \sup _{z \in \mathbf{R}^{d}} \int|\mathbf{E} g(t, z, \nu)| d t
\end{aligned}
$$

\section{Proof of Theorem 1.1}

In the proof of Theorem 1.1 we assume that $Q^{2}=I$, where $I$ is the identity operator, and, hence, that the symmetric operator $Q$ is isometric. 
This does not restrict the generality, since any symmetric operator $Q$ can be represented as $Q=Q_{+} Q_{0} Q_{+}$, where $Q_{+}$is a non-negative operator and $Q_{0}$ is a symmetric operator with $Q_{0}^{2}=I$. This can be derived, e.g., from $[16$, Section 7.5 , Theorem 1]. In general case one should apply the estimates proved below with replacing $Q$ by $Q_{0}, S_{N}-a$ by $Q_{+}\left(S_{N}-a\right), X$ by $Q_{+} X$, $\eta$ by $Q_{+} \eta$, etc.

Without loss of generality we assume that

$$
\sigma^{2}=\mathbf{E}|X|^{2}=c_{11}, \quad \text { and, hence, } \quad \mathbf{E}|X|^{8 / 3} \geqslant c,
$$

where $c_{11}$ is an absolute positive constant. Otherwise; one should consider the vectors $\left\{c_{11} \sigma^{-1} X_{1}, c_{11} \sigma^{-1} X_{2}, \ldots\right\}$ instead of $\left\{X_{1}, X_{2}, \ldots\right\}$. In principle, one can take $c_{11}=1$. However, in Section 4 we shall use the possibility to choose $c_{11}$ to be sufficiently small.

Suppose that the distribution

$$
H=H_{N}=\mathcal{L}\left(\frac{X}{\sqrt{N}}\right) \in \mathfrak{F}_{d}
$$

is represented in the form

$$
H=(1-q) U+q V,
$$

where $U=U_{N} \in \mathfrak{F}_{d}, V=V_{N} \in \mathfrak{F}_{d}$,

$$
U\left\{x \in \mathbf{R}^{d}:|x| \leqslant N^{1 / 4}\right\}=V\left\{x \in \mathbf{R}^{d}:|x|>N^{1 / 4}\right\}=1
$$

and

$$
0 \leqslant q=q_{N}=\mathbf{P}\left\{|X|>N^{3 / 4}\right\} \leqslant 1 .
$$

Set

$$
W=W_{N}=(1-q) U+q E .
$$

Introduce the truncated i.i.d. random vectors

$$
X_{j}^{*}=X_{j} I\left\{\left|X_{j}\right| \leqslant N^{3 / 4}\right\}, \quad j=1,2, \ldots,
$$

with

$$
\mathcal{L}\left(\frac{X_{j}^{*}}{\sqrt{N}}\right)=W, \quad j=1,2, \ldots
$$

and denote

$$
X_{j}^{* *}=X_{j}-X_{j}^{*}=X_{j} I\left\{\left|X_{j}\right|>N^{3 / 4}\right\}, \quad j=1,2, \ldots
$$

Obviously,

$$
\mathbf{E} X_{j}^{* *}+\mathbf{E} X_{j}^{*}=\mathbf{E} X_{j}=0, \quad j=1,2, \ldots .
$$

In order to simplify the notation it is convenient to suppose that the i.i.d. vectors $Y, Y_{1}, Y_{2}, \ldots$ are defined on the same probability space as $X, X_{1}$, $X_{2}, \ldots$ as follows. Assume that the random variable $\Pi$ and the random 
vectors $Y_{1}^{*}, Y_{2}^{*}, \ldots ; Y_{1}^{* *}, Y_{2}^{* *}, \ldots ; X, X_{1}, X_{2}, \ldots$ are mutually independent. Let $\Pi$ have the Poisson distribution with parameter 1 . Denote

$$
Y=X_{1}+\cdots+X_{\Pi}
$$

Assume that $Y_{1}^{*}, Y_{2}^{*}, \ldots ; Y_{1}^{* *}, Y_{2}^{* *}, \ldots$ have distributions such that

$$
\begin{aligned}
& \mathcal{L}\left(\frac{Y_{j}^{*}}{\sqrt{N}}\right)=e(W)=e((1-q) U) \\
& \mathcal{L}\left(\frac{Y_{j}^{* *}}{\sqrt{N}}\right)=e(q V), \quad j=1,2, \ldots
\end{aligned}
$$

Write

$$
\begin{gathered}
Y_{j}=Y_{j}^{*}+Y_{j}^{* *}, \quad j=1,2, \ldots, \\
S_{N}^{*}=N^{-1 / 2}\left(X_{1}^{*}+\cdots+X_{N}^{*}\right), \quad T_{N}^{*}=N^{-1 / 2}\left(Y_{1}^{*}+\cdots+Y_{N}^{*}\right) .
\end{gathered}
$$

Obviously, $Y_{1}, Y_{2}, \ldots$ are i.i.d. random vectors with

$$
\mathcal{L}\left(\frac{Y_{j}}{\sqrt{N}}\right)=e((1-q) U) e(q V)=e(H)=\mathcal{L}\left(\frac{Y}{\sqrt{N}}\right), \quad j=1,2, \ldots,
$$

and

$$
\begin{array}{ll}
\mathcal{L}\left(S_{N}\right)=H^{N}, & \mathcal{L}\left(T_{N}\right)=(e(H))^{N}=e(N H) \\
\mathcal{L}\left(S_{N}^{*}\right)=W^{N}, & \mathcal{L}\left(T_{N}^{*}\right)=(e(W))^{N}=e(N W)
\end{array}
$$

(see $(1.1)-(1.3),(1.6),(3.2),(3.3),(3.11)-(3.14))$. By construction, the vectors $Y_{1}, Y_{2}, \ldots$ are independent of $X, X_{1}, X_{2}, \ldots$ Note that (1.1), (3.2), (3.15) automatically imply that

$$
\mathcal{L}\left(Y_{j}\right)=\mathcal{L}(Y)=e(\mathcal{L}(X)), \quad: j=1,2, \ldots
$$

According to (1.7), (3.17),

$$
\mathbf{E} Y_{j}=\mathbf{E} X_{j}=\mathbf{E} \eta=0, \quad \operatorname{cov} Y_{j}=\operatorname{cov} X_{j}=\operatorname{cov} \eta, \quad j=1,2, \ldots
$$

Introduce also the symmetrized random vectors

$$
\begin{array}{ll}
\tilde{X}_{j}=X_{j}-X_{N+j}, & \tilde{Y}_{j}=Y_{j}-Y_{N+j}, \\
\tilde{X}_{j}^{*}=X_{j}^{*}-X_{N+j}^{*}, \quad \tilde{Y}_{j}^{*}=Y_{j}^{*}-Y_{N+j}^{*}, & j=1,2, \ldots
\end{array}
$$

It is clear that (3.18) and (3.19) imply that, for $j=1,2, \ldots$,

$$
\begin{aligned}
\mathbf{E}\left(\frac{\tilde{X}_{j}}{\sqrt{2}}\right) & =\mathbf{E}\left(\frac{\tilde{Y}_{j}}{\sqrt{2}}\right)=0, \\
\operatorname{cov}\left(\frac{\tilde{Y}_{j}}{\sqrt{2}}\right) & =\operatorname{cov}\left(\frac{\tilde{X}_{j}}{\sqrt{2}}\right)=\operatorname{cov} \eta,
\end{aligned}
$$


and

$$
\mathbf{E}\left|\frac{\tilde{X}_{j}}{\sqrt{2}}\right|^{8 / 3} \leqslant c \mathbf{E}|X|^{8 / 3} .
$$

Besides, using (3.11), (3.15), (3.19) and Lemma 2.3 for fixed values of $I$, we see that

$$
\begin{aligned}
\mathbf{E}\left|\frac{\tilde{Y}_{j}}{\sqrt{2}}\right|^{8 / 3} & \leqslant c \mathbf{E}|Y|^{8 / 3}=c \mathbf{E}\left|X_{1}+\cdots+X_{\Pi}\right|^{8 / 3} \\
& \leqslant c \mathbf{E} \Pi^{4 / 3} \mathbf{E}|X|^{8 / 3} \leqslant c \mathbf{E}|X|^{8 / 3} .
\end{aligned}
$$
we get

Using (1.6), (3.5), (3.7), (3.14), and applying the Chebyshev inequality,

$$
\mathbf{P}\left\{\left|S_{N}\right| \neq\left|S_{N}^{*}\right|\right\} \leqslant \sum_{j=1}^{N} \mathbf{P}\left\{X_{j} \not \equiv X_{j}^{*}\right\}=N q \leqslant \frac{\mathbf{E}|X|^{8 / 3}}{N} .
$$

Using (1.1), (1.6) and (3.12)-(3.14), we obtain

$$
\begin{aligned}
\mathbf{P}\left\{Y_{j} \neq Y_{j}^{*}\right\} & =\mathbf{P}\left\{Y_{j}^{* *} \neq 0\right\} \leqslant 1-e^{-q} \leqslant q \\
& \leqslant \frac{\mathbf{E}|X|^{8 / 3}}{N^{2}}, \quad j=1,2, \ldots, \\
\mathbf{P}\left\{\left|T_{N}\right| \neq\left|T_{N}^{*}\right|\right\} & \leqslant \sum_{j=1}^{N} \mathbf{P}\left\{Y_{j} \neq Y_{j}^{*}\right\} \leqslant \frac{\mathbf{E}|X|^{8 / 3}}{N} .
\end{aligned}
$$

Introduce the distribution functions

$$
\begin{aligned}
F(x) & =\mathbf{P}\left\{Q\left[S_{N}-a\right]<x\right\}, \\
G(x) & =\mathbf{P}\left\{Q\left[T_{N}-a\right]<x\right\}, \\
F^{*}(x) & =\mathbf{P}\left\{Q\left[S_{N}^{*}-a\right]<x\right\}, \\
G^{*}(x) & =\mathbf{P}\left\{Q\left[T_{N}^{*}-a\right]<x\right\},
\end{aligned}
$$

and the characteristic functions

$$
\begin{aligned}
f^{*}(t) & =\mathbf{E} \exp \left\{i t Q\left[S_{N}^{*}-a\right]\right\}, \\
g^{*}(t) & =\mathbf{E} \exp \left\{i t Q\left[T_{N}^{*}-a\right]\right\}, \quad t \in \mathbf{R} .
\end{aligned}
$$

From (3.23)-(3.25) it is easy to derive that

$$
\begin{aligned}
& \left|F(x)-F^{*}(x)\right| \leqslant P\left\{\left|S_{N}\right| \neq\left|S_{N}^{*}\right|\right\} \leqslant \frac{\mathbf{E}|X|^{8 / 3}}{N}, \quad \text { for all } x \in \mathbf{R} . \\
& \left|G(x)-G^{*}(x)\right| \leqslant P\left\{\left|T_{N}\right| \neq\left|T_{N}^{*}\right|\right\} \leqslant \frac{\mathbf{E}|X|^{8 / 3}}{N},
\end{aligned}
$$

Fix an orthonormal system of vectors $\mathcal{E}=\left\{e_{1}, \ldots, e_{9}\right\} \in \mathbf{R}^{d}$ and put

$$
\delta_{0}=\frac{1}{900}, \quad p=\min _{e \in \mathcal{E} \cup Q \mathcal{E}} P\left\{|\eta-e| \leqslant \delta_{0}\right\}
$$

4 Теория вероятностей и ее применения, № 2 
The number $p$ is positive since the Gaussian vector $\eta$ is not concentrated in any proper closed linear subspace of $\mathbf{R}^{d}$ and, therefore, $\mathbf{P}\{\eta \in B\}>0$, for any ball $B \subset \mathbf{R}^{d}$ with positive radius. According to the definition of the classes $\mathcal{N}(\cdot)$, we have

$$
\mathcal{L}(\eta) \in \mathcal{N}\left(9, p, \delta_{0}, Q ; e_{1}, \ldots, e_{9}\right) .
$$

It is clear that

$$
p \leqslant c \mathbf{E}|\eta|^{8 / 3} \leqslant c \mathbf{E}|X|^{8 / 3}
$$

(see (2.20), (3.28)). If $p$ is fixed, one can consider it as a function of the covariance operator $\operatorname{cov} X=\operatorname{cov} \eta$. The same applies to functions of $p$.

Below we shall show that

$$
\sup _{x}|F(x)-G(x)| \leqslant \frac{c\left(|a|^{4}+\left(\mathbf{E}|X|^{8 / 3}\right)^{3}\right)}{p^{4} N}
$$

provided that $\mathbf{E}|X|^{2}=c_{11}$, the operator $Q$ is isometric and $p$ is defined in (3.28).

Let $c_{12}$ be a sufficiently large constant to be specified later. Let $m$ be the minimal positive integer number such that $m \geqslant c_{12}\left(E|X|^{8 / 3}\right)^{3} / p^{3}$. In view of (3.30), we can choose $c_{12}$ to be so large that

$$
\frac{c_{12}\left(\mathrm{E}|X|^{8 / 3}\right)^{3}}{p^{3}} \leqslant m \leqslant \frac{2 c_{12}\left(\mathbf{E}|X|^{8 / 3}\right)^{3}}{p^{3}} .
$$

Write

$$
\begin{array}{ll}
K=\frac{c_{13} N}{m}, & N_{0}=p K=\frac{c_{13} p N}{m} \leqslant \frac{p N}{m} \\
t_{0}=\frac{N_{0}^{2 / 9}}{p}, & t_{1}=\frac{\sqrt{N_{0}}}{p}, \quad t_{2}=\frac{c_{14} N_{0}^{7 / 9}}{p},
\end{array}
$$

where $c_{13} \leqslant 1$ will be chosen to be sufficiently small and $c_{14}$ sufficiently large. From (3.32), (3.33) it follows that

$$
\frac{c_{15} p^{4} N}{2\left(\mathrm{E}|X|^{8 / 3}\right)^{3}} \leqslant N_{0} \leqslant \frac{c_{15} p^{4} N}{\left(\mathrm{E}|X|^{8 / 3}\right)^{3}}
$$

Without loss of generality (see (3.28), (3.30)-(3.33), (3.35)) we can assume that

$$
1 \leqslant m \leqslant \frac{N}{20}, \quad p N m^{-1} \geqslant c_{16}, \quad N_{0} \geqslant c_{17},
$$

where $c_{16}=c_{16}\left(c_{12}\right), c_{17}=c_{17}\left(c_{12}, c_{13}, c_{14}, c_{16}\right)$ will be chosen to be sufficiently large. We have

$$
0<t_{0}<t_{1}<t_{2}<K,
$$


if $c_{17}$ in (3.36) is large enough. The function $M_{9}\left(p t, N_{0}\right)$ attains its minimal value at the point $t=t_{1}$ and, according to (2.2),

$$
M_{9}\left(p t, N_{0}\right)=\left\{\begin{array}{lll}
|p t|^{-9 / 2}, & \text { for } & |t| \leqslant t_{1}, \\
\left(\frac{|p t|}{N_{0}}\right)^{9 / 2}, & \text { for } & |t| \geqslant t_{1}
\end{array}\right.
$$

Introduce the integral

$$
I\left(f^{*}, g^{*}\right)=\int_{|t| \leqslant t_{0}}\left|\frac{f^{*}(t)-g^{*}(t)}{t}\right| d t .
$$

Applying Lemma 2.6 with $H_{1}=F^{*}, H_{2}=G^{*}, T=K$, splitting the integrals and using (3.37) and that $1 / K \leqslant 1 /|t|$, for $|t| \leqslant K$, we obtain

$$
\left|F^{*}(x)-G^{*}(x)\right| \leqslant c I\left(f^{*}, g^{*}\right)+c \sum_{k=1}^{4}\left(I_{k}\left(f^{*}\right)+I_{k}\left(g^{*}\right)\right),
$$

where we denote

$$
\begin{array}{ll}
I_{1}\left(f^{*}\right)=\frac{1}{K} \int_{|t| \leqslant t_{0}}\left|f^{*}(t)\right| d t, & I_{2}\left(f^{*}\right)=\int_{t_{0} \leqslant|t| \leqslant t_{1}}\left|f^{*}(t)\right| \frac{d t}{|t|}, \\
I_{3}\left(f^{*}\right)=\int_{t_{1} \leqslant|t| \leqslant t_{2}}\left|f^{*}(t)\right| \frac{d t}{|t|}, & I_{4}\left(f^{*}\right)=\int_{t_{2} \leqslant|t| \leqslant K}\left|f^{*}(t)\right| \frac{d t}{|t|} .
\end{array}
$$

Below we shall show that each of the integrals $I_{k}\left(f^{*}\right), k=1, \ldots, 4$, is bounded from above by $c / N_{0}$.

Define the functions

$$
\begin{aligned}
& R_{1}(t)=\sup _{b \in \mathbf{R}^{d}}\left|\mathrm{E} \exp \left\{i t^{*} Q\left[S_{N_{1}}^{*}-b\right]\right\}\right|, \\
& R_{2}(t)=\sup _{b \in \mathbf{R}^{d}}\left|\mathrm{E} \exp \left\{i t^{*} Q\left[T_{N_{1}}^{*}-b\right]\right\}\right|,
\end{aligned}
$$

where

$$
\frac{N}{20} \leqslant N_{1} \stackrel{\text { def }}{=}\left\lceil\frac{N-1}{10}\right\rceil \leqslant \frac{N}{10}
$$

(see (3.36)). To estimate the integrals $I_{1}, I_{2}$ and $I_{3}$ we note that the sums $S_{N}^{*}, S_{N_{1}}^{*}$ can be represented as

$$
S_{N}^{*}=\left(\frac{N_{1}}{N}\right)^{1 / 2} S_{N_{1}}^{*}+b_{1}, \quad S_{N_{1}}^{*}=\left(\frac{2 m}{N_{1}}\right)^{1 / 2} \Upsilon_{n}^{*}+b_{2},
$$

where the random vectors $\Upsilon_{n}^{*}$ can be written in the form

$$
\Upsilon_{n}^{*}=Z_{1}^{*}+\cdots+Z_{n}^{*}, \quad n=\left\lceil\frac{N_{1}}{m}\right\rceil
$$


$Z_{1}^{*}, \ldots, Z_{n}^{*}$ are independent copies of $Z^{*}$ defined by

$$
Z^{*}=(2 m)^{-1 / 2}\left(X_{1}^{*}+\cdots+X_{m}^{*}\right)
$$

and $b_{1}, b_{2}$ are independent of $S_{N_{1}}^{*}, \Upsilon_{n}^{*}$ respectively. Furthermore, introduce the symmetrized random vectors (see (3.19))

$$
\begin{aligned}
\tilde{Z} & =m^{-1 / 2}\left(\frac{\widetilde{X}_{1}}{\sqrt{2}}+\cdots+\frac{\widetilde{X}_{m}}{\sqrt{2}}\right) \quad \text { and } \\
\widetilde{Z}^{*} & =m^{-1 / 2}\left(\frac{\tilde{X}_{1}^{*}}{\sqrt{2}}+\cdots+\frac{\widetilde{X}_{m}^{*}}{\sqrt{2}}\right) .
\end{aligned}
$$

According to (3.36), (3.43) and (3.45), we have

$$
\frac{N}{40 m} \leqslant n \leqslant \frac{N}{10 m} \text {. }
$$

Let $e \in \mathbf{R}^{d},|e|=1$. Consider a function $L: \mathbf{R}^{d} \rightarrow \mathbf{R}$ with infinitely many bounded derivatives such that

$$
0 \leqslant L(\cdot) \leqslant 1, \quad L(x)=1, \quad \text { for }|x| \leqslant 1, \quad L(x)=0, \quad \text { for } \quad|x| \geqslant 2 .
$$

Using (3.21), (3.47)-(3.49) and applying Lemma 2.4, we have

$$
\mathbf{P}\left\{|\widetilde{Z}-e| \leqslant 2 \delta_{0}\right\} \geqslant \mathrm{E} L\left(\frac{\widetilde{Z}-e}{\delta_{0}}\right) \geqslant \mathbf{E} L\left(\frac{\eta-e}{\delta_{0}}\right)-\frac{c \mathbf{E}|X|^{8 / 3}}{m^{1 / 3} \delta_{0}^{8 / 3}} .
$$

From (3.49) it follows that

$$
\mathbf{E} L\left(\frac{\eta-e}{\delta_{0}}\right) \geqslant \mathbf{P}\left\{|\eta-e| \leqslant \delta_{0}\right\} .
$$

It is evident that

$$
\mathbf{P}\left\{\left|\tilde{Z}^{*}-e\right| \leqslant 2 \delta_{0}\right\} \geqslant \mathbf{P}\left\{|\tilde{Z}-e| \leqslant 2 \delta_{0}\right\}-\mathbf{P}\left\{\tilde{Z} \neq \tilde{Z}^{*}\right\} .
$$

According to (3.5), (3.7), (3.19), (3.24) and (3.36), we have

$$
\mathbf{P}\left\{\widetilde{Z}_{\not} \not \tilde{Z}^{*}\right\} \leqslant \sum_{j=1}^{m} \mathbb{P}\left\{\widetilde{X}_{j} \neq \tilde{X}_{j}^{*}\right\} \leqslant 2 m q \leqslant \frac{2 m \mathbf{E}|X|^{8 / 3}}{N^{2}} \leqslant \frac{2 \mathbf{E}|X|^{8 / 3}}{m^{1 / 3}}
$$

Applying (3.50)-(3.53) for $e=e_{j}$ and $e=Q e_{j}, j=1, \ldots, 9$, choosing $c_{12}$ in (3.32) to be large enough and using (3.28), we obtain

$$
2 \mathbf{P}\left\{\left|\widetilde{Z}^{*}-e_{j}\right| \leqslant 2 \delta_{0}\right\} \geqslant 2 \mathbf{P}\left\{\left|\eta-e_{j}\right| \leqslant \delta_{0}\right\}-\frac{c \mathbf{E}|X|^{8 / 3}}{m^{1 / 3}} \geqslant 2 p-p=p
$$

and, analogously,

$$
2 \mathrm{P}\left\{\left|\widetilde{Z}^{*}-Q e_{j}\right| \leqslant 2 \delta_{0}\right\} \geqslant p .
$$


According to (2.1), the relations (3.54), (3.55) imply that

$$
\mathcal{L}\left(\widetilde{Z}^{*}\right) \in \mathcal{N}\left(9, \frac{p}{2}, 2 \delta_{0}, Q ; e_{1}, \ldots, e_{9}\right) .
$$

Taking into account relations $(3.28),(3.44),(3.45),(3.56)$, we see that Lemma 2.1 is applicable with $s=9, \delta=2 \delta_{0}, r=p / 2 ; y_{j}=e_{j}, j=1, \ldots, 9$.

Conditioning on $b_{2}$ (see (2.26)), applying (2.11) of Lemma 2.1 with $u=2 t m N^{-1} n$, and taking into account (2.4), (3.33), (3.42), (3.44), (3.48), we obtain that, for any $b \in \mathbf{R}^{d}, t \in \mathbf{R}$,

$$
\begin{aligned}
& \left|\mathbf{E} \exp \left\{i t^{*} Q\left[S_{N_{1}}^{*}-b\right]\right\}\right| \\
& \quad=\left|\mathbf{E} \exp \left\{2 i t m N^{-1} Q\left[\Upsilon_{n}^{*}+\left(\frac{2 m}{N_{1}}\right)^{-1 / 2}\left(b_{2}-b\right)\right]\right\}\right| \\
& \quad \leqslant \sup _{z \in \mathbf{R}^{d}}\left|\mathbf{E} \exp \left\{2 i t m N^{-1} Q\left[\Upsilon_{n}^{*}-z\right]\right\}\right| \\
& \quad \leqslant c M_{9}\left(p t m N^{-1} n, \frac{p n}{2}\right) \leqslant c M_{9}\left(p t, N_{0}\right)
\end{aligned}
$$

Note that in the last inequality we used (2.4) to change constants in the arguments of the function $M_{9}$.

Using (2.26) again, we see that the relations (3.26), (3.42), (3.44), (3.57) together imply that, for any $t \in \mathbf{R}$,

$$
\left|f^{*}(t)\right| \leqslant R_{1}(t) \leqslant c \min \left\{1, M_{9}\left(p t, N_{0}\right)\right\} .
$$

Using (3.34), (3.37), (3.38), (3.41) and (3.58), we conclude

$$
I_{1}\left(f^{*}\right) \leqslant \frac{c}{K} \int_{0}^{1 / p} d t+\frac{c}{K} \int_{1 / p}^{\infty} \frac{d t}{(p t)^{9 / 2}}=\frac{c}{p K}=\frac{c}{N_{0}}
$$

Similarly,

$$
I_{2}\left(f^{*}\right) \leqslant c \int_{t_{0}}^{t_{1}} \frac{1}{(p t)^{9 / 2}} \frac{d t}{t} \leqslant c \int_{p t_{0}}^{\infty} \frac{d t}{t^{1+9 / 2}}=\frac{c}{N_{0}}
$$

and

$$
I_{3}\left(f^{*}\right) \leqslant c \int_{0}^{t_{2}}\left(\frac{p t}{N_{0}}\right)^{9 / 2} \frac{d t}{t} \leqslant c\left(\frac{p t_{2}}{N_{0}}\right)^{9 / 2}=\frac{c}{N_{0}} .
$$

Using (2.27), (3.26), (3.33), (3.34), (3.41), (3.44), (3.45), (3.48), (3.56), applying the inequality (2.7) of Lemma 2.1 and changing variables, we obtain

$$
I_{4}\left(f^{*}\right) \leqslant c N_{0}^{-2} \log N_{0}+\sup _{\Lambda} \sup _{z \in \mathbf{R}^{d}} \int_{t_{2} \leqslant|t| \leqslant K} \sqrt{\varphi_{\Lambda}\left(z, c m t l N^{-1}\right)} \frac{d t}{|t|}
$$


with $\varphi_{\Lambda}(\cdot, \cdot)$ defined by $(2.8)$ and

$$
l=\left\lceil\frac{r n}{36}\right\rceil=\left\lceil\frac{p n}{72}\right\rceil \leqslant c N_{0}
$$

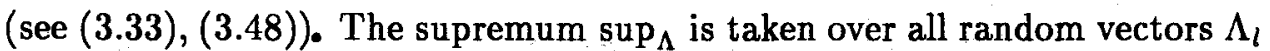
and $\Lambda_{l}^{\prime}$ of type (2.9), where $W_{1}, \ldots, W_{l}, W_{1}^{\prime}, \ldots, W_{l}^{\prime} \in \mathbf{R}^{d}$ denote independent random vectors with distributions from the class $\Gamma\left(9,2 \delta_{0} ; e_{1}, \ldots, e_{9}\right)$. In addition, we used that, in view of (3.33), (3.48), (3.63), we can choose $c_{16}, c_{17}$ in (3.36) to be so large that

$$
l=\left\lceil\frac{p n}{72}\right\rceil \geqslant\left\lceil\frac{p N}{2880 m}\right\rceil \geqslant c N_{0} \geqslant 1 .
$$

Changing variables, using (3.33), (3.34), (3.63), (3.64) and choosing appropriate constants $c_{13}, c_{14}$ in the definitions of $t_{2}$ and $K$, we have

$$
\int_{t_{2} \leqslant|t| \leqslant K} \sqrt{\varphi_{\Lambda}(z, c m t l N-1)} \frac{d t}{|t|} \leqslant \int_{N_{0}^{7 / 9} \leqslant|u| \leqslant N_{0}} \sqrt{\varphi_{\Lambda}(z, u)} \frac{d u}{|u|} .
$$

Applying inequality (2.10) of Lemma 2.1 and using (2.4), (3.63) and (3.64), we obtain, for all $\gamma \geqslant 0, u \in \mathbf{R}$,

$$
\varphi_{\Lambda}(z, u+\gamma) \varphi_{\Lambda}(z, u-\gamma) \leqslant c M_{9}^{2}(\gamma, l) \leqslant c_{18} M_{9}^{2}\left(2 \gamma, N_{0}\right) .
$$

Therefore, Lemma 2.2 is applicable with $\psi(t)=\varphi_{\Lambda}(z, t)$ and $\psi(t)=\varphi_{\Lambda}(z,-t)$; $s=9, D=N_{0}, \lambda=\sqrt{c_{18}}$. By Lemma 2.2, we have

$$
\int_{N_{0}^{7 / 9} \leqslant|u| \leqslant N_{0}} \sqrt{\varphi_{\Lambda}(z, u)} \frac{d u}{|u|} \leqslant \frac{c}{N_{0}} .
$$

Using (3.62), (3.65), (3.67), we see that $I_{4}\left(f^{*}\right) \leqslant c / N_{0}$ and, taking into account (3.59)-(3.61),

$$
\left|I_{1}\left(f^{*}\right)+I_{2}\left(f^{*}\right)+I_{3}\left(f^{*}\right)+I_{4}\left(f^{*}\right)\right| \leqslant \frac{c}{N_{0}} .
$$

Similarly to (3.58), (3.68), one proves

$$
\begin{aligned}
& \left|g^{*}(t)\right| \leqslant R_{2}(t) \leqslant c \min \left\{1, M_{9}\left(p t, N_{0}\right)\right\}, \\
& \left|I_{1}\left(g^{*}\right)+I_{2}\left(g^{*}\right)+I_{3}\left(g^{*}\right)+I_{4}\left(g^{*}\right)\right| \leqslant \frac{c}{N_{0}} .
\end{aligned}
$$

In order to verify these relations one should use (3.25), (3.26), (3.41), (3.42) and the fact that, according to (1.6), (3.14), (3.15), (3.18), (3.20), (3.22), (3.24), the random vectors $Y, Y_{1}, Y_{2}, \ldots ; Y_{1}^{*}, Y_{2}^{*}, \ldots ; T_{N}, T_{N}^{*}$ have all the properties of the random vectors $X, X_{1}, X_{2}, \ldots ; X_{1}^{*}, X_{2}^{*}, \ldots ; S_{N}, S_{N}^{*}$, which were used in the proof of (3.58), (3.68). 
In order to estimate $I\left(f^{*}, g^{*}\right)$ we write the characteristic functions $f^{*}(t)$ and $g^{*}(t)$ in the form

$$
\begin{aligned}
f^{*}(t) & =\int_{\mathbf{R}^{d}} \exp \{\text { it } Q[x-a]\} W^{N}\{d x\} \\
g^{*}(t) & =\int_{\mathbf{R}^{d}} \exp \{i t Q[x-a]\}(e(W))^{N}\{d x\}
\end{aligned}
$$

(see (3.16), (3.26)). It is clear that

$$
\begin{aligned}
W^{N}-(e(W))^{N} & =(W-e(W)) \sum_{j=1}^{N} W^{j-1}(e(W))^{N-j} \\
e(W) & =\exp \{W-E\}=E+\sum_{\mu=1}^{\infty} \frac{(W-E)^{\mu}}{\mu !}
\end{aligned}
$$

and, hence,

$$
\begin{aligned}
W-e(W) & =-\sum_{\mu=2}^{\infty} \frac{(W-E)^{\mu}}{\mu !}=-(W-E)^{2} \sum_{\mu=0}^{\infty} \frac{(W-E)^{\mu}}{(\mu+2) !} \\
& =(W-E)^{2} \sum_{\mu=0}^{\infty} \sum_{\nu=0}^{\mu} \alpha_{\mu, \nu} W^{\nu}
\end{aligned}
$$

where $\alpha_{\mu, \nu}$ are easily computable coefficients with

$$
\sum_{\nu=0}^{\mu}\left|\alpha_{\mu, \nu}\right|=\frac{2^{\mu}}{(\mu+2) !}, \quad \mu=0,1, \ldots
$$

According to (3.71), (3.72), (3.74),

$$
\begin{aligned}
f^{*}(t)-g^{*}(t)= & \sum_{j=1}^{N} \sum_{\mu=0}^{\infty} \sum_{\nu=0}^{\mu} \alpha_{\mu, \nu} J(t, j, \nu, N) \\
= & \sum_{j=1}^{N} \sum_{\mu=0}^{N} \sum_{\nu=0}^{\mu} \alpha_{\mu, \nu} J(t, j, \nu, N) \\
& +\sum_{j=1}^{N} \sum_{\mu=N+1}^{\infty} \sum_{\nu=0}^{\mu} \alpha_{\mu, \nu} J(t, j, \nu, N) \\
= & \sum_{1} \alpha_{\mu, \nu} J(t, j, \nu, N)+\sum_{2} \alpha_{\mu, \nu} J(t, j, \nu, N)
\end{aligned}
$$

where

$$
\begin{aligned}
J(t, j, \nu, N)= & \int_{\mathbf{R}^{d}} \exp \{i t Q[x-a]\} \\
& \times\left((W-E)^{2} W^{j-1+\nu}(e(W))^{N-j}\right)\{d x\}
\end{aligned}
$$


It is easy to see that, for $j=1, \ldots, N ; \mu=1,2, \ldots ; \nu=0, \ldots, \mu$, the integral $J(t, j, \nu, N)$ can be rewritten in the form

$$
\begin{aligned}
J(t, j, \nu, N)= & \mathbf{E} \exp \left\{i t Q\left[\xi+\zeta_{1}^{*}+\zeta_{2}^{*}\right]\right\}-\mathbf{E} \exp \left\{i t Q\left[\xi+\zeta_{1}^{*}\right]\right\} \\
& -\mathbf{E} \exp \left\{i t Q\left[\xi+\zeta_{2}^{*}\right]\right\}+\mathbf{E} \exp \{i t Q[\xi]\},
\end{aligned}
$$

where

$$
\zeta_{k}^{*}=\zeta_{k} \mathbf{I}\left\{\left|\zeta_{k}\right| \leqslant N^{1 / 4}\right\}, \quad k=1,2,
$$

and the random vectors $\zeta_{1}, \zeta_{2} \in \mathbf{R}^{d}$ with distributions

$$
\mathcal{L}\left(\zeta_{1}\right)=\mathcal{L}\left(\zeta_{2}\right)=H=\mathcal{L}\left(\frac{X}{\sqrt{N}}\right)
$$

form together with $X_{1}^{*}, X_{2}^{*}, \ldots ; Y_{1}^{*}, Y_{2}^{*}, \ldots$ a system of mutually independent random vectors such that

$$
\begin{aligned}
\xi & =N^{-1 / 2}\left(X_{1}^{*}+\cdots+X_{j-1+\nu}^{*}+Y_{1}^{*}+\cdots+Y_{N-j}^{*}\right)-a, \\
\mathcal{L}(\xi) & =W^{j-1+\nu}(e(W))^{N-j} E_{-a}
\end{aligned}
$$

(see (3.8), (3.12)). Here we used that (3.7), (3.8), (3.79), (3.80) together imply that

$$
\mathcal{L}\left(\zeta_{1}^{*}\right)=\mathcal{L}\left(\zeta_{2}^{*}\right)=W=\mathcal{L}\left(\frac{X_{1}^{*}}{\sqrt{N}}\right)
$$

Furthermore, we introduce random vectors $\zeta_{k}^{* *}$ by

$$
\zeta_{k}^{* *}=\zeta_{k}-\zeta_{k}^{*}=\zeta_{k} \mathrm{I}\left\{\left|\zeta_{k}\right|>N^{1 / 4}\right\}, \quad k=1,2 .
$$

According to (3.80) and (3.83),

$$
\mathbf{E} \zeta_{k}^{* *}+\mathbf{E} \zeta_{k}^{*}=\mathbf{E} \zeta_{k}=0, \quad k=1,2 .
$$

For fixed values of $\xi$, we apply Lemma 2.5 to the right-hand side of (3.78). Taking the expectation with respect to $\xi$, we see that

$$
J(t, j, \nu, N)=J_{1}+J_{2},
$$

where $J_{1}, J_{2}$ are defined by $(2.24)$, (2.25) with $v_{1}, v_{2}, w_{1}, w_{2}, u$ satisfying (2.22) and with $\tau_{1}, \tau_{2}$ independent of $\left\{\zeta_{1}^{*}, \zeta_{2}^{*} ; X_{1}^{*}, X_{2}^{*}, \ldots ; Y_{1}^{*}, Y_{2}^{*}, \ldots\right\}$ and uniformly distributed on the interval $[0,1]$.

It can be shown that

$$
\begin{aligned}
\left|J_{1}\right| \leqslant & c\left(t^{2}+t^{4}\right) \mathbf{E}\left|\zeta_{1}^{*}\right|^{2}\left(D_{1}^{(\nu)} \mathbf{E}\left|\zeta_{1}^{*}\right|^{2}+D_{2}^{(\nu)} \mathbf{E}\left|\zeta_{1}^{*}\right|^{4}\right)\left(R_{1}(t)+R_{2}(t)\right) \\
\left|J_{2}\right| \leqslant & c\left(|t|+|t|^{3}\right) \mathbf{E}\left|\zeta_{1}^{* *}\right|\left(\mathbf{E}\left|\zeta_{1}^{* *}\right|+\mathbf{E}\left|\zeta_{1}^{*}\right|^{2}+\mathbf{E}\left|\zeta_{1}^{*}\right|^{3}\right) \\
& \times D_{1}^{(\nu)}\left(R_{1}(t)+R_{2}(t)\right)
\end{aligned}
$$


with

$$
\begin{aligned}
D_{1}^{(\nu)} & =1+|a|^{4}+\sup _{1 \leqslant \rho \leqslant N_{2}} \frac{\mathbf{E}\left|X_{1}^{*}+\cdots+X_{\rho}^{*}\right|^{4}+\mathbf{E}\left|Y_{1}^{*}+\cdots+Y_{\rho}^{*}\right|^{4}}{N^{2}}, \\
D_{2}^{(\nu)} & =1+|a|^{2}+\sup _{1 \leqslant \rho \leqslant N_{2}} \frac{\left.\mathbf{E}\left|X_{1}^{*}+\cdots+X_{\rho}^{*}\right|^{2}+\mathbf{E}\left|Y_{1}^{*}+\cdots+Y_{\rho}^{*}\right|^{2}\right)}{N}, \\
N_{2} & =N_{2}(\nu)=\left\lceil\frac{N+\nu-1}{10}\right\rceil \geqslant N_{1}
\end{aligned}
$$

(see (3.42), (3.43)). To justify this bounds we note that, according to (3.81), the random variable $\xi$ can be represented in the form

$$
\xi=\xi_{0}+\xi_{1}+\xi_{2}+\cdots+\xi_{10}+\xi_{11},
$$

where $\xi_{0}, \xi_{1}, \xi_{2}, \ldots, \xi_{10}, \xi_{11}$ are independent random vectors which are independent of $\tau_{1}, \tau_{2}, \zeta_{1}^{*}, \zeta_{2}^{*}$ and

$$
\begin{aligned}
\xi_{0} & =-a ; \quad \mathcal{L}\left(\xi_{1}\right)=\mathcal{L}\left(x_{1}^{(1)}+\cdots+x_{k}^{(1)}\right), \quad \text { for some } \quad k \leqslant N_{2} ; \\
\mathcal{L}\left(\xi_{l}\right) & =\mathcal{L}\left(x_{1}^{(l)}+\cdots+x_{N_{2}}^{(l)}\right), \quad l=2, \ldots, 11 ; \\
\mathcal{L}\left(x_{m}^{(l)}\right) & =\mathcal{L}\left(\frac{X_{1}^{*}}{\sqrt{N}}\right) \quad \text { or } \\
\mathcal{L}\left(x_{m}^{(l)}\right) & =\mathcal{L}\left(\frac{Y_{1}^{*}}{\sqrt{N}}\right), \quad m=1, \ldots, N_{2}, \quad l=1, \ldots, 11,
\end{aligned}
$$

and, moreover, at least 5 of $\xi_{l}$ are identically distributed with

$$
\begin{aligned}
& \mathcal{L}\left(\xi_{l}\right)=\mathcal{L}\left(N^{-1 / 2}\left(X_{1}^{*}+\cdots+X_{N_{2}}^{*}\right)\right) \quad \text { or } \\
& \mathcal{L}\left(\xi_{l}\right)=\mathcal{L}\left(N^{-1 / 2}\left(Y_{1}^{*}+\cdots+Y_{N_{2}}^{*}\right)\right) .
\end{aligned}
$$

To prove (3.86) one should separately estimate each summand on the right-hand side of (2.24). For example, using (2.22), (3.89), we see that

$$
\begin{gathered}
\mathbf{E} v_{1}^{2} w_{1}^{2}\left(1-\tau_{1}\right)\left(1-\tau_{2}\right) \exp \left\{\tau_{2} v_{1}+\tau_{1} w_{1}\right\} \exp \{i t Q[\xi]\} \\
=16 t^{4} \sum_{l_{1}, l_{2}, l_{3}, l_{4}=0}^{11} \mathbf{E} A\left(l_{1}, l_{2}, l_{3}, l_{4}\right) B
\end{gathered}
$$

where

$$
\begin{aligned}
A\left(l_{1}, l_{2}, l_{3}, l_{4}\right) & \\
= & \left\langle Q \xi_{l_{1}}, \zeta_{1}^{*}\right\rangle\left\langle Q \xi_{l_{2}}, \zeta_{1}^{*}\right\rangle\left\langle Q \xi_{l_{3}}, \zeta_{2}^{*}\right\rangle\left\langle Q \xi_{l_{4}}, \zeta_{2}^{*}\right\rangle\left(1-\tau_{1}\right)\left(1-\tau_{2}\right), \\
B= & \exp \left\{2 i t\left\langle Q \xi_{0}+\cdots+Q \xi_{11}, \tau_{2} \zeta_{1}^{*}+\tau_{1} \zeta_{2}^{*}\right\rangle\right. \\
& \left.+i t Q\left[\xi_{0}+\cdots+\xi_{11}\right]\right\} .
\end{aligned}
$$


Consider a summand from the right-hand side of (3.92) with fixed $l_{1}, l_{2}, l_{3}, l_{4}$. It is clear that at least one of the variables $\xi_{l}, l=1, \ldots, 11$, say $\xi_{l_{0}}$, has the distribution (3.91) and is independent of $\xi_{l_{1}}, \xi_{l_{2}}, \xi_{l_{3}}, \xi_{l_{4}}$ and, of course, of $\tau_{1}, \tau_{2}, \zeta_{1}^{*}, \zeta_{2}^{*}$. The same independence properties are satisfied for the summands composing $\xi_{l_{0}}$. Denote by $\mathbf{E}_{0}$ the conditional expectation with respect to some $N_{1}$ of the $N_{2}$ summands composing $\xi_{i_{0}}$ given all the remaining variables and vectors. According to (2.26), (3.14), (3.42), (3.88), (3.91), (3.93),

$$
\left|\mathrm{E}_{0} B\right| \leqslant R_{1}(t)+R_{2}(t) \quad \text { a.s. }
$$

Using the relations $|\langle x, y\rangle| \leqslant|x| \cdot|y|,(3.82),(3.88),(3.90),(3.93)$, the isometry property of $Q$ and Hölder's inequality, we have

$$
\mathrm{E}\left|A\left(l_{1}, l_{2}, l_{3}, l_{4}\right)\right| \leqslant\left(\mathrm{E}\left|\zeta_{1}^{*}\right|^{2}\right)^{2} D_{1}^{(\nu)} .
$$

By virtue of (3.94), (3.95),

$$
\begin{aligned}
\left|\mathbf{E} A\left(l_{1}, l_{2}, l_{3}, l_{4}\right) B\right| & =\left|\mathbf{E}\left(A\left(l_{1}, l_{2}, l_{3}, l_{4}\right) \mathbf{E}_{0} B\right)\right| \leqslant \mathbf{E}\left(\left|A\left(l_{1}, l_{2}, l_{3}, l_{4}\right)\right|\left|\mathbf{E}_{0} B\right|\right) \\
& \leqslant \mathbf{E}\left|A\left(l_{1}, l_{2}, l_{3}, l_{4}\right)\right|\left(R_{1}(t)+R_{2}(t)\right) \\
& \leqslant\left(\mathbf{E}\left|\zeta_{1}^{*}\right|^{2}\right)^{2} D_{1}^{(\nu)}\left(R_{1}(t)+R_{2}(t)\right)
\end{aligned}
$$

Therefore, the left-hand side of (3.92) can be estimated in absolute value by the right-hand side of (3.86). The estimation of the remaining 6 summands on the right-hand side of $(2.24)$ can be done in a similar way. Here the elementary inequality $x^{y} \leqslant 1+x^{z}$, for $x>0,0 \leqslant y \leqslant z$, has to be used several times.

The proof of (3.87) almost coincides with the proof of (3.86). A unique difference consists in changing $\zeta_{k}^{*}$ (involving in the corresponding summand of (2.25) in one place only) by $-\zeta_{k}^{* *}$ (see $\left.(2.22),(2.25),(3.84)\right)$. Notice that these $\zeta_{k}^{*}, \zeta_{k}^{* *}$ are independent of all other random variables and vectors in the expression of this summand. For example, using (2.22), (3.79), (3.81), (3.83), (3.84), we see that the vectors $\zeta_{1}, \zeta_{1}^{*}, \zeta_{1}^{* *}$ are independent of $\xi, \zeta_{2}^{*}, \tau_{1}$ and

$$
\begin{aligned}
\mathbf{E} v_{1} & w_{1}^{2}\left(1-\tau_{1}\right) \exp \left\{\tau_{1} w_{1}\right) \exp \{i t Q[\xi]\} \\
= & -8 i t^{3} \mathbf{E}\left\langle Q \xi, \zeta_{1}^{*}\right\rangle\left\langle Q \xi, \zeta_{2}^{*}\right\rangle^{2}\left(1-\tau_{1}\right) \exp \left\{2 i t \tau_{1}\left\langle Q \xi, \zeta_{2}^{*}\right\rangle\right\} \exp \{i t Q[\xi]\} \\
= & 8 i t^{3} \mathbf{E}\left\langle Q \xi, \zeta_{1}^{* *}\right\rangle\left\langle Q \xi, \zeta_{2}^{*}\right)^{2}\left(1-\tau_{1}\right) \\
& \times \exp \left\{2 i t \tau_{1}\left\langle Q \xi, \zeta_{2}^{*}\right\rangle\right\} \exp \{i t Q[\xi]\}
\end{aligned}
$$

The further estimation of this quantity is similar to the estimation of the right-hand side of (3.92). One should also use that $\zeta_{1}^{* *}, \zeta_{2}^{* *}$ are identically distributed and, hence, $\mathbf{E}\left|\zeta_{2}^{* *}\right|=\mathbf{E}\left|\zeta_{1}^{* *}\right|$. 
Consider the estimation of the right-hand sides of (3.86), (3.87). According to (3.1), (3.9), (3.79), (3.80), (3.83), we have

$$
\begin{aligned}
& \mathbf{E}\left|\zeta_{1}^{*}\right|^{2} \leqslant c N^{-1}, \quad \mathbf{E}\left|\zeta_{1}^{*}\right|^{3} \leqslant c N^{-3 / 4}, \quad \mathbf{E}\left|\zeta_{1}^{*}\right|^{4} \leqslant N^{-1} \mathbf{E}|X|^{8 / 3}, \\
& \mathbf{E}\left|\zeta_{1}^{* *}\right| \leqslant N^{-5 / 4} \mathbf{E}\left|X_{1}^{* *}\right|^{2} \leqslant c N^{-5 / 4} .
\end{aligned}
$$

Let $y \stackrel{\text { def }}{=} \mathbf{E} X_{1}^{*}$. In view of $(1.1),(3.8),(3.12)$, it is easy to verify that

$$
\mathbf{E} Y_{k}^{*}=\mathbf{E} X_{k}^{*}=y, \quad \operatorname{cov} Y_{k}^{*}=\operatorname{cov} X_{k}^{*}=\operatorname{cov} X_{1}^{*}, \quad k=1,2 \ldots
$$

Introduce the centered random vectors $\bar{X}_{k}^{*}, \bar{Y}_{k}^{*}$ by

$$
\bar{X}_{k}^{*}=X_{k}^{*}-y, \quad \bar{Y}_{k}^{*}=Y_{k}^{*}-y, \quad k=1,2 \ldots
$$

Using (3.101), we see that, for $\rho=1,2, \ldots$,

$$
\begin{aligned}
& \mathbf{E}\left|X_{1}^{*}+\cdots+X_{\rho}^{*}\right|^{4} \leqslant c\left(\mathbf{E}\left|\bar{X}_{1}^{*}+\cdots+\bar{X}_{\rho}^{*}\right|^{4}+\rho^{4}|y|^{4}\right) \\
& \mathbf{E}\left|Y_{1}^{*}+\cdots+Y_{\rho}^{*}\right|^{4} \leqslant c\left(\mathbf{E}\left|\bar{Y}_{1}^{*}+\cdots+\bar{Y}_{\rho}^{*}\right|^{4}+\rho^{4}|y|^{4}\right) \\
& \mathbf{E}\left|X_{1}^{*}+\cdots+X_{\rho}^{*}\right|^{2} \leqslant c\left(\rho \mathbf{E}\left|\bar{X}_{1}^{*}\right|^{2}+\rho^{2}|y|^{2}\right) \\
& \mathbf{E}\left|Y_{1}^{*}+\cdots+Y_{\rho}^{*}\right|^{2} \leqslant c\left(\rho \mathbf{E}\left|\bar{Y}_{1}^{*}\right|^{2}+\rho^{2}|y|^{2}\right) .
\end{aligned}
$$

Using Lemma 2.3, Hölder's inequality and the relations (3.100), (3.101), we obtain that

$$
\begin{aligned}
\mathbf{E}\left|\bar{X}_{1}^{*}+\cdots+\bar{X}_{\rho}^{*}\right|^{4} & \leqslant c\left(\rho \mathbf{E}\left|\bar{X}_{1}^{*}\right|^{4}+\rho^{2}\left(\mathbf{E}\left|\bar{X}_{1}^{*}\right|^{2}\right)^{2}\right) \\
& \leqslant c\left(\rho\left(\mathbf{E}\left|X_{1}^{*}\right|^{4}+|y|^{4}\right)+\rho^{2}\left(\mathbf{E}\left|X_{1}^{*}\right|^{2}+|y|^{2}\right)^{2}\right) \\
& \leqslant c\left(\rho \mathbf{E}\left|X_{1}^{*}\right|^{4}+\rho^{2}\left(\mathbf{E}\left|X_{1}^{*}\right|^{2}\right)^{2}\right)
\end{aligned}
$$

and, analogously,

$$
\mathrm{E}\left|\bar{Y}_{1}^{*}+\cdots+\bar{Y}_{\rho}^{*}\right|^{4} \leqslant c\left(\rho \mathbf{E}\left|Y_{1}^{*}\right|^{4}+\rho^{2}\left(\mathbf{E}\left|Y_{1}^{*}\right|^{2}\right)^{2}\right) .
$$

According to (3.1), (3.7), (3.100), (3.101),

$$
\mathbf{E}\left|\bar{Y}_{1}^{*}\right|^{2}=\mathbf{E}\left|\bar{X}_{1}^{*}\right|^{2} \leqslant 4 \mathbf{E}\left|X_{1}^{*}\right|^{2} \leqslant c, \quad \mathbf{E}\left|X_{1}^{*}\right|^{4} \leqslant N \mathbf{E}|X|^{8 / 3} .
$$

Recall that $I$ denotes an independent of $X_{1}^{*}, X_{2}^{*}, \ldots$ random variable with Poisson distribution of parameter 1. In view of the relations (1.1), (3.8), (3.12), we obtain $\mathcal{L}\left(Y_{1}^{*}\right)=\mathcal{L}\left(X_{1}^{*}+\cdots+X_{\Pi}^{*}\right)$. Therefore, for $\beta>0$,

$$
\begin{aligned}
\mathbf{E}\left|Y_{1}^{*}\right|^{\beta} & =\mathbf{E}\left|X_{1}^{*}+\cdots+X_{\Pi}^{*}\right|^{\beta} \leqslant \mathbf{E \Pi}^{\beta}\left(\left|X_{1}^{*}\right|^{\beta}+\cdots+\left|X_{\Pi}^{*}\right|^{\beta}\right) \\
& =\mathbf{E E}\left\{\Pi^{\beta}\left(\left|X_{1}^{*}\right|^{\beta}+\cdots+\left|X_{\Pi}^{*}\right|^{\beta}\right) \mid \Pi\right\} \\
& =\mathbf{E}\left|X_{1}^{*}\right|^{\beta} \mathbf{E} \Pi^{\beta+1}=\boldsymbol{c}(\beta) \mathbf{E}\left|X_{1}^{*}\right|^{\beta} .
\end{aligned}
$$


Using (3.1), (3.9), (3.10), (3.100), we see that

$$
|y|=\left|\mathbf{E} X_{1}^{* *}\right| \leqslant \mathbf{E}\left|X_{1}^{* *}\right| \leqslant c N^{-3 / 4} .
$$

From (3.88), (3.102)-(3.107) it follows that

$$
\begin{aligned}
& D_{1}^{(\nu)} \leqslant 1+|a|^{4}+c\left(N_{2} N^{-1} \mathbf{E}|X|^{8 / 3}+N_{2}^{2} N^{-2}+N_{2}^{4} N^{-5}\right), \\
& D_{2}^{(\nu)} \leqslant 1+|a|^{2}+c\left(N_{2} N^{-1}+N_{2}^{2} N^{-5 / 2}\right) .
\end{aligned}
$$

Recall that in the sum $\sum_{1}$ from (3.76) we have

$$
0 \leqslant \nu \leqslant \mu \leqslant N, \quad N_{2} \leqslant \frac{N}{5}
$$

(see (3.88)). Therefore, (3.108) and (3.109) together imply

$$
D_{1}^{(\nu)} \leqslant c\left(1+|a|^{4}+\mathbf{E}|X|^{8 / 3}\right), \quad D_{2}^{(\nu)} \leqslant c\left(1+|a|^{2}\right) .
$$

Using (3.1), (3.58), (3.69), (3.85)-(3.87), (3.98), (3.99) and (3.110), we infer that the integrals $J(t, j, \nu, N)$ from $\sum_{1}$ can be estimated in absolute value as follows:

$$
|J(t, j, \nu, N)| \leqslant c C N^{-2}\left(|t|+t^{4}\right) \min \left\{1, M_{9}\left(p t, N_{0}\right)\right\},
$$

where

$$
C=\left(|a|^{4}+\left(1+|a|^{2}\right) \mathbf{E}|X|^{8 / 3}\right) .
$$

Analogously, for the sum $\sum_{2}$ from (3.76) we have

$$
0 \leqslant \max \{\nu, N\} \leqslant \mu, \quad N_{2} \leqslant \frac{\mu}{5}
$$

(see (3.88)). In this case (3.108) and (3.113) imply

$$
D_{1}^{(\nu)} \leqslant c \mu^{4}\left(1+|a|^{4}+\mathrm{E}|X|^{8 / 3}\right), \quad D_{2}^{(\nu)} \leqslant c \mu^{2}\left(1+|a|^{2}\right) .
$$

Using (3.114) instead of (3.110), we obtain for the integrals $J(t, j, \nu, N)$ from $\sum_{2}$ the following analog of (3.111):

$$
|J(t, j, \nu, N)| \leqslant c C \mu^{4} N^{-2}\left(|t|+t^{4}\right) \min \left\{1, M_{9}\left(p t, N_{0}\right)\right\} .
$$

From (3.75), (3.76), (3.111) and (3.115) it follows that, for all $t \in \mathbf{R}$,

$$
\left|f^{*}(t)-g^{*}(t)\right| \leqslant c C N^{-1}\left(|t|+t^{4}\right) \min \left\{1, M_{9}\left(p t, N_{0}\right)\right\} .
$$

Using (3.28), (3.37), (3.38), we see that

$$
\int_{|t| \leqslant t_{0}}\left(1+|t|^{3}\right) \min \left\{1, M_{9}\left(p t, N_{0}\right)\right\} d t=2 \int_{0}^{1 / p}+2 \int_{1 / p}^{t_{0}} \leqslant \frac{c}{p^{4}} .
$$


In view of $(3.39),(3.116)$ and $(3.117)$, we have

$$
\left|I\left(f^{*}, g^{*}\right)\right| \leqslant \frac{c C}{p^{4} N} .
$$

Now the statement of Theorem 1.1 follows from $(3.25),(3.27),(3.30),(3.35)$, (3.40), (3.68), (3.70) and (3.118). Moreover, we have in fact proved the following explicit bound

$$
\sup _{x}|F(x)-G(x)| \leqslant \frac{c\left(C+\left(\mathbf{E}|X|^{8 / 3}\right)^{3}\right)}{p^{4} N} .
$$

It is clear that $(3.1),(3.112),(3.119)$ imply (3.31).

\section{An explicit bound}

Under additional restrictions an explicit bound for the constant in (1.10) is given in Theorem 4.1. Below we assume that the conditions of Theorem 1.1 are satisfied and $Q^{2}=I$. Let $\mathbb{E}$ be the set of eigenvectors of the operator $\operatorname{cov} X=\operatorname{cov} \eta$. Denote by $\mathcal{Z}=\left\{z_{1}, z_{2} \ldots\right\} \subset \mathbb{E}$ an orthonormal basis which diagonalizes the operator $\operatorname{cov} \eta$ and such that $\sigma_{1}^{2}=\mathbf{E}\left(\eta, z_{1}\right)^{2} \geqslant \sigma_{2}^{2}=$ $\mathbf{E}\left\langle\eta, z_{2}\right\rangle^{2} \geqslant \ldots$ are the ordered eigenvalues of this operator. Suppose that there exist at least 9 different $z_{j} \in \mathcal{Z}$ such that $Q z_{j} \in \mathbb{E}$. Note that $\left|Q z_{j}\right|=1$ for all $z_{j} \in \mathcal{Z}$ since the operator $Q$ is isometric. Define

$$
\sigma_{9}^{2}(Q, \mathcal{Z})=\sup _{\mathcal{E}} \min _{e \in \mathcal{E} \cup Q \mathcal{E}} \mathbf{E}\langle\eta, e\rangle^{2},
$$

where $\sup _{\mathcal{E}}$ is taken over all orthonormal systems of vectors $\mathcal{E}=\left\{y_{1}, \ldots\right.$, $\left.y_{9}\right\} \subset \mathcal{Z}$ such that $Q \mathcal{E} \subset \mathbb{E}$. Note that if the operator $Q$ is diagonal in the basis $z_{1}, z_{2} \ldots$, then $\sigma_{9}^{2}(Q, \mathcal{Z})=\sigma_{9}^{2}$.

Theorem 4.1. Let the conditions described above be satisfied. Then

$$
\begin{gathered}
\sup _{x}\left|\mathbf{P}\left\{Q\left[S_{N}-a\right]<x\right\}-\mathbf{P}\left\{Q\left[T_{N}-a\right]<x\right\}\right| \\
\leqslant \frac{1}{N}\left(\frac{\left(\mathbf{E}|X|^{8 / 3}\right)^{3}}{\sigma^{8}}+\frac{|a|^{4}}{\sigma^{4}}\right) \exp \left\{\frac{c \sigma^{2}}{\sigma_{9}^{2}(Q, \mathcal{Z})}\right\},
\end{gathered}
$$

where $\sigma^{2}=\mathbf{E}|X|^{2}$.

$\mathrm{P}$ r o o f. Without loss of generality we assume that $\sigma^{2}=\mathbf{E}|X|^{2}=c_{11}$ (see (3.1)). Hence, inequality (3.31) is valid with $p$ defined in (3.28). Below we show that the vectors $e_{1}, \ldots, e_{9}$ can be chosen so that

$$
p \geqslant \exp \left\{-\frac{c}{\sigma_{9}^{2}(Q, \mathcal{Z})}\right\} \text {. }
$$

Let $e \in \mathbf{E}$ and $|e|=1$. Define $\sigma_{e}^{2}=\mathbf{E}\langle\eta, e\rangle^{2}$. According to (3.28), (4.1), it suffices to verify that

$$
\mathbf{P}\left\{|\eta-e| \leqslant \delta_{0}\right\} \geqslant \exp \left\{-c \sigma_{e}^{-2}\right\} .
$$


Introduce the Gaussian vectors $\eta-\langle\eta, e\rangle e$ and $\langle\eta, e\rangle e$. These vectors are independent, since $e \in \mathbb{E}$. Consequently,

$$
\begin{aligned}
\mathbf{P}\left\{|\eta-e| \leqslant \delta_{0}\right\} \geqslant & \mathbf{P}\left\{|\eta-\langle\eta, e\rangle e|+|\langle\eta, e\rangle e-e| \leqslant \delta_{0}\right\} \\
\geqslant & \mathbf{P}\left\{|\eta-\langle\eta, e\rangle e| \leqslant \frac{\delta_{0}}{2}\right\} \\
& \times \mathbf{P}\left\{|\langle\eta, e\rangle e-e| \leqslant \frac{\delta_{0}}{2}\right\} \stackrel{\text { def }}{=} p_{1} \cdot p_{2} .
\end{aligned}
$$

Recall that $\delta_{0}=\frac{1}{900}$. Therefore, we have

$$
\begin{aligned}
p_{1} & =1-\mathbf{P}\left\{|\eta-\langle\eta, e\rangle e|^{2} \geqslant \frac{\delta_{0}^{2}}{4}\right\} \geqslant 1-c \mathbf{E}|\eta-\langle\eta, e\rangle|^{2} \\
& \geqslant 1-c \mathbf{E}|\eta|^{2}=1-c \mathbf{E}|X|^{2} \geqslant \frac{1}{2}
\end{aligned}
$$

and

$$
\sigma_{e}^{2}=\mathbf{E}(\eta, e)^{2} \leqslant \mathbf{E}|\eta|^{2}=\mathbf{E}|X|^{2} \leqslant 1
$$

provided that $c_{11}$ in (3.1) is chosen to be sufficiently small. It remains to estimate $p_{2}$. It is easy to see that

$$
p_{2}=\mathbf{P}\left\{\left|\eta_{0}-\sigma_{e}^{-1}\right| \leqslant \frac{\delta_{0}}{2 \sigma_{e}}\right\},
$$

where $\eta_{0}$ is a random variable with standard normal distribution. Taking into account that $\delta_{0}=\frac{1}{900}$, we see that the relations (4.5)-(4.8) imply (4.4) and, hence, (4.3).

Now inequality (4.2) follows from (3.1), (3.25), (3.31), (4.3).

\section{REFERENCES}

1. de Acosta $A$. Inequalities for $B$-valued random vectors with applications to the strong law of large numbers. - Ann. Probab., 1981, v. 9, p. 157-161.

2. Арак T. В. О сближении $n$-кратных сверток распределений, имеющих неотрицательную характеристичесхую функцию, с сопровождающими законами. - Теория вероятн. и ее примен., 1980, т. XXV, в. 2, с. 225-246.

3. Арак T. В. О скорости сходимости в равномерной предельной теореме Колмогорова. I; II. - Теория вероятн. и ее примен., 1981, т. XXVI, в. 2, с. 225-245; в. 3, c. $449-463$.

4. Арак Т. В. Уточнение нижней ощенки для скорости сходимости в равномерной предельной теореме Колмогорова. - Теория вероятн. и ее примен., 1982, т. XXVII, в. 4, с. 767-772.

5. Арак Т. В., Зайчев $А$. Ю. Равномерные предельные теоремы для сумм независимых случайных величин. - Труды Матем. ин-та им. В. А. Стехлова АН СССР, 1986, т. 174 , с. $1-214$. 
6. Bakštys G., Paulauskas V. J. Approximation of distributions of sums of Banachvalued random elements with infinitely divisible laws. I; II. - Lithuanian Math. J., 1986 , v. 26, № 3 , p. 207-215; 1987, v. 27, № 2, p. 106-113.

7. Беяткус В., Гётие Ф., Паулаускас B., Рачкаускас $A$. Точность гауссовской аппроксимации в банаховых пространствах. - Итоги науки и техники. Сер. Совр. пробл. матем., 1991, т. 81, с. 39-139.

8. Bentkus V., Götze F. On the lattice point problem for ellipsoids. - Preprint 94-111 SFB 343, Universität Bielefeld, 1994 (to appear in Acta Arithmetica).

9. Bentkus $V$., Götze $F$. Optimal rates of convergence in functional limit theorems for quadratic forms. - Preprint 95-094 SFB 343, Universität Bielefeld, 1995.

10. Чекаяавичус B. Аппроксимация сопровождающими распределениями и асимптотические разложения. І. - Литов. матем. сб., 1989, т. 29, № 1, с. 171-178.

11. Esseen C.-G. On the concentration function of a sum of independent random variables. - Z. Wahrscheinlichkeitstheor. verw. Geb., 1968, B. 9, S. 290-308.

12. Ибрагимов И. А., Пресмая Э. Л. О скорости сближения распределений сумм независимых случайных величин с сопровождающими законами. - Теория вероятн. и ее примен., 1973, т. XVIII, в. 4, с. 753-766.

13. Колмогоров $A$. H. Некоторые работы последних лет в области предельных теорем теории вероятностей. - Вестник МГУ, 1953, т. 10, с. 29-38.

14. Колмогоров $A$. $H$. Две равномерные предельные теоремы для сумм независимых слагаемых. - Теория вероятн. и ее примен., 1956, т. I, в. 4, с. 426-436.

15. Le Cam L. On the distribution of sums of independent random variables. - Bernoulli, Bayes, Laplace (anniversary volume). Berlin-Heidelberg: Springer-Verlag, 1965, p. 179-202.

16. Люстерник Л. А., Соболев В. И. Элементы функционального анализа. М.: Наука, $1965,520 \mathrm{c}$.

17. Nagaev $S$. $V$. Concentration functions and approximation with infinitely divisible laws in Hilbert space. - Preprint 90-094 SFB 343, Universität Bielefeld, 1990.

18. Петров В. В. Суммы независимых случайных величин. М.: Наука, 1972, 416 с.

19. Пресман Э. Л. О многомерном варианте равномерной предельной теоремы Колмогорова. - Теория вероятн. и ее примен., 1973, т. XVIII, в. 2, с. 396-402.

20. Прохоров Ю. В. Суммы одинаково распределенных случайных величин. - Докл. AH CCCP, 1955 , т. 105 , в. 2, с. $645-647$.

21. Зайчев $A$. $Ю$. Некоторые свойства $n$-кратных сверток распределений. - Теория вероятн. и ее примен., 1981, т. XXVI, в. 1, с. 152-156.

22. Zaitsev A. Yu. Estimates for the closeness of successive convolutions of multidimensional symmetric distributions. - Probab. Theory Relat. Fields, 1988, v. 79, № 2, p. $175-200$.

23. Зайчев $A$. $О$. Об аппроксимации сверток многомерных симметричных распределений сопровождающими законами. - Записки научн. семин. ЛОМИ, 1989, т. 177 , с. $55-72$.

24. Зайчев $A$. Ю. Об одном классе неравномерных оценок в многомерных предельных теоремах. - Записки научн. семин. ЛОМИ, 1990, т. 184, с. 92-105.

25. Зайцев $A$. Ю. Аппроксимащия сверток сопровождающими законами при условии существования моментов низшего порядка. - Записки научн. семин. ПІОМИ, 1996, т. 228. 